Groups Geom. Dyn. 7 (2013), 931-959

DOI $10.4171 / \mathrm{GGD} / 211$
Groups, Geometry, and Dynamics

(C) European Mathematical Society

\title{
Bredon cohomological finiteness conditions for generalisations of Thompson groups
}

\author{
Conchita Martínez-Pérez and Brita E. A. Nucinkis*
}

\begin{abstract}
We define a family of groups that generalises Thompson's groups $T$ and $G$, and also those of Higman, Stein and Brin. For groups in this family we describe centralisers of finite subgroups and show that for a given finite subgroup $Q$ there are finitely many conjugacy classes of finite subgroups isomorphic to $Q$. We consider groups of type quasi-F $\infty$. This is a property slightly weaker than possessing a finite type model for the classifying space of proper actions $\underline{E} G$. We give criteria for the $T$-versions of our groups to be of type quasi- $\underline{F} \infty$. We also generalise some well-known properties of ordinary cohomology to Bredon cohomology.
\end{abstract}

Mathematics Subject Classification (2010). 20J05.

Keywords. Bredon cohomology, Thompson groups, finiteness properties.

\section{Introduction}

Thompson's groups $F, T$ and $G$ (also denoted by $V$ ), which can be defined as certain homeomorphism groups of the unit interval, the circle and the Cantor-set, respectively, have received a large amount of attention in recent years. There are many interesting generalisations of these groups, such as the Higman-Thompson groups $F_{n, r}, T_{n, r}$, $G_{n, r}$ (recall that $T=T_{2,1}$ and $G=G_{2,1}$ ), the $T$ - and $G$-groups defined by Stein [24] and the higher dimensional Thompson groups $s V=s G_{2,1}$ defined by Brin [4]. All these groups contain free abelian groups of infinite rank, are finitely presented and with the exception of $s V$ for $s \geq 4$ are known to be of type $\mathrm{FP}_{\infty}$ ([5], [24], [12], [9]). ${ }^{1}$ Furthermore, the $G$ - and $T$-groups contain finite groups of arbitrarily large orders. In this paper we consider automorphism groups of certain Cantor algebras which include Higman-Thompson, Stein and Brin's groups.

As in the original exposition by Higman [10] and in Brown's proof [5] that $F_{n, r}$, $T_{n, r}$ and $G_{n, r}$ are of type $\mathrm{FP}_{\infty}$, we consider a Cantor algebra $U_{r}(\Sigma)$ on a so-called

\footnotetext{
*The first named author was partially supported by BFM2010-19938-C03-03, Gobierno de Aragón and European Union's ERDF funds.

${ }^{1}$ Note added in proof. The groups $s V$ have now been shown to be of type $\mathrm{FP}_{\infty}$ by Fluch, Schwandt, Witzel and Zaremsky, arXiv:1207.4832.
} 
valid set of relations $\Sigma$ and define groups $G_{r}(\Sigma)$ as follows: the elements of $G_{r}(\Sigma)$ are bijections between certain subsets of $U_{r}(\Sigma)$, which we call admissible. One can show that these groups are finitely generated, see [18]. Provided that the relations in $\Sigma$ are order-preserving we can also define the groups $T_{r}(\Sigma)$, which are given by cyclic order-preserving bijections. One can also define generalisations of $F_{n, r}$.

The admissible subsets of $U_{r}(\Sigma)$ form a poset, and the groups $T_{r}(\Sigma)$ and $G_{r}(\Sigma)$ act on the geometric realisation $\left|\mathfrak{A}_{r}(\Sigma)\right|$ of this poset (for the original HigmanThompson algebras this was already used by Brown in [5]).

Let $G$ be either $T_{r}(\Sigma)$ or $G_{r}(\Sigma)$. For every finite subgroup $Q$ we consider the fixed point sets $\mathfrak{A}_{r}(\Sigma) Q$. The $Q$-set structure of every admissible subset $Y \in \mathfrak{A}_{r}(\Sigma)^{Q}$ is determined by its decomposition into transitive $Q$-sets. We show (Theorem 4.3) that there are finitely many conjugacy classes in $G$ of subgroups isomorphic to $Q$. Furthermore we show (Theorem 4.4) that there is an extension

$$
K \succ C_{G_{r}(\Sigma)}(Q) \rightarrow G_{r_{1}}(\Sigma) \times \cdots \times G_{r_{t}}(\Sigma)
$$

with locally finite kernel, where the $r_{1}, \ldots, r_{t}$ are integers uniquely determined by $Q$. We also get the analogous result (Theorem 4.8) for the groups $T_{r}(\Sigma)$ (if defined) that, for a certain $l$ also determined by $Q$, there is a central extension

$$
Q \succ C_{T_{r}(\Sigma)}(Q) \rightarrow T_{l}(\Sigma) .
$$

This generalises a result of Matucci [19], Theorem 7.1.5, for the original Thompson group $T$.

Recently a variant of the Eilenberg-Mac Lane space, the classifying space with respect to a family $\mathfrak{X}$ of subgroups, has been well researched. Let $X$ be a $G-C W$ complex. $X$ is said to be a model for $E_{\mathfrak{X}} G$, the classifying space with isotropy in the family $\mathfrak{X}$ if $X^{K}$ is contractible for $K \in \mathfrak{X}$ and $X^{K}$ is empty otherwise. The classifying space $X$ for a family satisfies the following universal property: whenever there is a $G$-CW-complex $Y$ with isotropy lying in the family $\mathfrak{X}$, there is a $G$-map $Y \rightarrow X$, which is unique up to $G$-homotopy. In particular, $E_{\mathfrak{X}} G$ is unique up to $G$-homotopy equivalence.

For the family $\mathfrak{F}$ of finite subgroups we denote $E_{\mathfrak{F}} G$ by $\underline{E} G$, the classifying space for proper actions. We say a group is of type $\underline{F}_{\infty}$ if it admits a finite type model for $\underline{E} G$. We show:

Theorem 3.1. $\left|\mathfrak{A}_{r}(\Sigma)\right|$ is a model for $\underline{E} G_{r}(\Sigma)$.

Obviously, this model has infinite dimension. Since these groups contain free abelian groups of infinite rank, they cannot possess any finite dimensional model. Exactly as ordinary classifying spaces yield free resolutions which can be used to define ordinary group cohomology, classifying spaces with isotropy in a family produce free resolutions in a functor category, which are used to define Bredon cohomology. We shall review properties of Bredon cohomology in Section 2. Many notions from 
ordinary cohomology have a Bredon analogue. For example, we say a group $G$ is of type Bredon-FP $\mathrm{F}_{\infty}$ if there is a Bredon-projective resolution of the constant Bredonmodule $\mathbb{Z}(-)$ by finitely generated Bredon-projective modules. The connection to classifying spaces and to ordinary cohomology is given by the following two results:

Theorem 1.1 ([17], Theorem 0.1). A group $G$ has a finite type model for a classifying space with isotropy in a family if and only if the group is of type Bredon-FP $\mathrm{P}_{\infty}$ and there is a model for the classifying space with finite 2-skeleton.

In particular we say a group is of type $\underline{\mathrm{FP}}_{\infty}$ if it is of type Bredon- $\mathrm{FP}_{\infty}$ for the family of finite subgroups.

Theorem 1.2 ([16], Theorem 4.2). A group $G$ admits a finite type model for $\underline{E} G$ if and only $G$ has finitely many conjugacy classes of finite subgroups and for each finite subgroup $K$ of $G$ the centraliser $C_{G}(K)$ is of type $\mathrm{FP}_{\infty}$ and finitely presented.

Equivalently, $G$ admits a finite type model for $\underline{E} G$ if and only it is of type $\underline{\mathrm{FP}}_{\infty}$ and centralisers of finite subgroups are finitely presented. The purpose of this paper is to study the possible finiteness conditions a model for $\underline{E} G$ for the groups $G_{r}(\Sigma)$ and $T_{r}(\Sigma)$ can satisfy. Since the groups we are considering do not have a bound on the orders of their finite subgroups, we need to weaken the condition on the number of conjugacy classes. We consider the property quasi-FP $\underline{\mathrm{FP}}_{\infty}$, which has the same condition on the centralisers of finite subgroups as $\underline{\mathrm{FP}}_{\infty}$ but just requires that for each finite subgroup $Q$ of $G$, there are only finitely many conjugacy classes of subgroups isomorphic to $Q$. Note that for groups with a bound on the orders of their finite subgroups both properties coincide. In [14] it was shown that there are examples of groups of type $\mathrm{FP}_{\infty}$, which have a bound on the orders of the finite subgroups, yet are not of type $\underline{\mathrm{FP}}_{\infty}$. These examples are virtually torsion free, admit a finite dimensional model for $\underline{E} G$ and can be constructed to have either infinitely many conjugacy classes of finite subgroups, or to have centralisers of finite subgroups not of type $\mathrm{FP}_{\infty}$. There are a number of classes of groups of type $\mathrm{FP}_{\infty}$ admitting cocompact models for $\underline{E} G$ including Gromov hyperbolic groups ([20]), Out $\left(F_{n}\right)$ ([25]) or elementary amenable groups of type $\mathrm{FP}_{\infty}([13])$.

Using our results on centralisers and conjugacy classes of finite subgroups we show:

Theorem 7.2. $T_{r}(\Sigma)$ is of type quasi- $\underline{\mathrm{FP}}_{\infty}$ if and only if $T_{l}(\Sigma)$ is of type $\mathrm{FP}_{\infty}$ for any $1 \leq l \leq d$ such that $\operatorname{gcd}(l, d) \mid r$.

We also consider the geometric analogue, to be of type quasi- $\underline{F}_{\infty}$, and the corresponding version of Theorem 7.2. We conjecture that similar results hold true for the groups $G_{r}(\Sigma)$.

The paper is structured as follows: In Section 2 we define the Cantor algebras and the corresponding generalisations of Thompson's groups $G$ and $T$. We then use 
this Cantor algebra to build a model for $\underline{E} G$ in Section 3. In Section 4 we prove the results on centralisers and conjugacy classes of finite subgroups that will be used later.

In Section 5 we collect all necessary background on Bredon cohomology with respect to an arbitrary family, and on Bredon cohomological finiteness conditions for modules. We prove an analogue to the Bieri-Eckmann criterion for property $\mathrm{FP}_{n}$ for modules. In Section 6 we specialise to the case of the family of finite subgroups and define what it means for a group to be quasi- $\underline{\mathrm{FP}}_{\infty}$ and quasi- $\underline{\mathrm{F}}_{\infty}$. Finally, the main results are proven in Section 7.

Acknowledgements. The authors wish to thank D. H. Kochloukova for very fruitful discussions, without which, in fact, this work probably would not have happened. We also thank F. Matucci for a conversation, which led us to discover a gap in a previous version of this paper.

\section{Generalisations of Higman-Thompson groups}

As mentioned in the introduction, the generalised Higman-Thompson groups can be viewed as certain automorphisms groups of Cantor algebras. We shall begin by defining these algebras. We use the notation of [12], Section 2. In particular, we consider a finite set $\{1, \ldots, s\}$ whose elements are called colours. To each colour $i$ we associate an integer $n_{i}>1$ which is called its arity. We say that $U$ is an $\Omega$-algebra if, for each colour $i$, the following operations (we let all operations act on the right) are defined in $U$ (for detail, see [7] and [12]):

i) One $n_{i}$-ary operation $\lambda_{i}$,

$$
\lambda_{i}: U^{n_{i}} \rightarrow U .
$$

We call these operations ascending operations or contractions.

ii) $n_{i} 1$-ary operations $\alpha_{i}^{1}, \ldots, \alpha_{i}^{n_{i}}$,

$$
\alpha_{i}^{j}: U \rightarrow U
$$

We call these operations 1-ary descending operations.

We write $\Omega=\left\{\lambda_{i}, \alpha_{i}^{j}\right\}_{i, j}$. For each colour $i$ we also consider the map $\alpha_{i}: U \rightarrow$ $U^{n_{i}}$ given by

$$
v \alpha_{i}:=\left(v \alpha_{i}^{1}, v \alpha_{i}^{2}, \ldots, v \alpha_{i}^{n_{i}}\right)
$$

for any $v \in U$. These maps are called descending operations or expansions. For any subset $Y$ of $U$, a simple expansion of colour $i$ of $Y$ is obtained by substituting some element $y \in Y$ by the $n_{i}$ elements of the tuple $y \alpha_{i}$. A simple contraction of colour $i$ of $Y$ is the set obtained by substituting a certain collection of $n_{i}$ distinct elements of $Y$, say $\left\{a_{1}, \ldots, a_{n_{i}}\right\}$, by $\left(a_{1}, \ldots, a_{n_{i}}\right) \lambda_{i}$. We also use the term operation to refer to the effect of a simple expansion, respectively contraction on a set. 
For any set $X$ there is an $\Omega$-algebra, free on $X$, which is called the $\Omega$-word algebra on $X$ and is denoted by $W_{\Omega}(X)$. An admissible subset $A \subseteq W_{\Omega}(X)$ is a subset that can be obtained after finitely many expansions or contractions from the set $X$.

Descending operations can be visualised by tree diagrams, see the following example with $X=\{x\}, s=1$ and $n_{1}=2$.

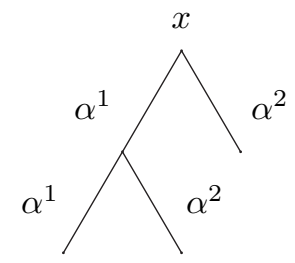

The set $A=\left\{x \alpha^{1} \alpha^{1}, x \alpha^{1} \alpha^{2}, x \alpha^{2}\right\}$ is an admissible subset. In pictures we often omit the maps and label the nodes by positive integers as follows.

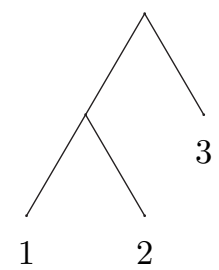

From now on we fix the set $X$ and assume it is finite. We consider the variety of $\Omega$-algebras satisfying a certain set of identities as follows.

Definition 2.1. Let $\Sigma$ be the following set of laws in the alphabet $X$.

i) For any $u \in W_{\Omega}(X)$, any colour $i$, and any $n_{i}$-tuple $\left(u_{1}, \ldots, u_{n_{i}}\right) \in W_{\Omega}(X)^{n_{i}}$,

$$
u \alpha_{i} \lambda_{i}=u, \quad\left(u_{1}, \ldots, u_{n_{i}}\right) \lambda_{i} \alpha_{i}=\left(u_{1}, \ldots, u_{n_{i}}\right) .
$$

The set of all these relations is denoted by $\Sigma_{1}$.

ii) A certain set

$$
\Sigma_{2}=\bigcup_{1 \leq i<i^{\prime} \leq s} \Sigma_{2}^{i, i^{\prime}}
$$

such that each $\Sigma_{2}^{i, i^{\prime}}$ is either empty or consists of all the laws of the following form: Consider first $i$ and fix a map $f:\left\{1, \ldots, n_{i}\right\} \rightarrow\{1, \ldots, s\}$. For each $1 \leq j \leq n_{i}$, we regard $\alpha_{i}^{j} \alpha_{f(j)}$ as a set of length 2 sequences of descending operations and let $\Lambda_{i}=\cup_{j=1}^{n_{i}} \alpha_{i}^{j} \alpha_{f(j)}$. Do the same for $i^{\prime}$ (with a corresponding map $f^{\prime}$ ) to get $\Lambda_{i^{\prime}}$ and now fix a bijection $\phi: \Lambda_{i} \rightarrow \Lambda_{i^{\prime}}$. Then $\Sigma_{2}^{i, i^{\prime}}$ is the set of laws

$$
u v=u \phi(v), \quad v \in \Lambda_{i}, u \in W_{\Omega}(X)
$$


(Note that by an abuse of notation we omit the $u \in W_{\Omega}(X)$ when we specify $\Sigma_{2}$ in the examples below.)

When factoring out the fully invariant congruence $\mathfrak{q}$ generated by $\Sigma$, we obtain an $\Omega$-algebra $W_{\Omega}(X) / \mathfrak{q}$ satisfying the identities in $\Sigma$. For details of the construction the reader is referred to [12], Section 2.

Definition 2.2. Let $r=|X|$ and $\Sigma$ as in Definition 2.1. Then the algebra $W_{\Omega}(X) / \mathfrak{q}=$ $U_{r}(\Sigma)$ is called a Cantor algebra.

Moreover, there is an epimorphism of $\Omega$-algebras

$$
W_{\Omega}(X) \rightarrow U_{r}(\Sigma), \quad A \mapsto \bar{A} .
$$

As in [12] we say that $\Sigma$ is valid if for any admissible $Y \subseteq W_{\Omega}(X)$, we have $|Y|=|\bar{Y}|$. This condition implies that $U_{r}(\Sigma)$ is a free object on $X$ in the class of those $\Omega$-algebras which satisfy the identities $\Sigma$ above.

If the set $\Sigma$ used to define $U_{r}(\Sigma)$ is valid, we also say that $U_{r}(\Sigma)$ is valid.

Example 2.3. Higman [10] defined an algebra $V_{n, r}$ with $|X|=r, s=1$ and arity $n$ as above with $\Sigma_{2}$ being empty. This algebra, which we call Higman algebra, is used in the original construction of the Higman-Thompson-groups $G_{n, r}$. For details see also [5]. In particular, these algebras are valid ([10], Section 2).

Example 2.4. Higman's construction for arity $n=2$ can be generalised as follows ([12], Section 2): Let $s \geq 1$ and $n_{i}=2$ for all $1 \leq i \leq s$. Hence we consider the set of $s$ colours $\{1, \ldots, s\}$, all of which have arity 2, together with the relations $\Sigma:=\Sigma_{1} \cup \Sigma_{2}$ with

$$
\Sigma_{2}:=\left\{\alpha_{i}^{l} \alpha_{j}^{t}=\alpha_{j}^{t} \alpha_{i}^{l} \mid 1 \leq i \neq j \leq s, l, t=1,2\right\} .
$$

Then $\Sigma$ is valid (see [12], Lemma 2.9).

Furthermore one can also consider $s$ colours, all of arity $n_{i}=n$, for all $1 \leq i \leq s$. Let

$$
\Sigma_{2}:=\left\{\alpha_{i}^{l} \alpha_{j}^{t}=\alpha_{j}^{t} \alpha_{i}^{l} \mid 1 \leq i \neq j \leq s, 1 \leq l, t \leq n\right\} .
$$

Using the same arguments as in [12], Section 2, one can show that the $\Sigma$ obtained in this way is also valid.

We call the resulting Cantor algebras $U_{r}(\Sigma)$ Brin algebras.

The tree diagram below visualises the relations in $\Sigma_{2}$. Here $r=1, s=2$ and $n=2$. We express an expansion of colour 1 with dotted lines and an expansion of colour 2 by solid lines. The leaves with the same label are identified.

Definition 2.5. Let $\Sigma$ be valid and consider $Y, Z \subseteq U_{r}(\Sigma)$. If $Z$ can be obtained from $Y$ by a finite number of simple expansions then we say that $Z$ is a descendant of $Y$ and write

$$
Y \leq Z \text {. }
$$



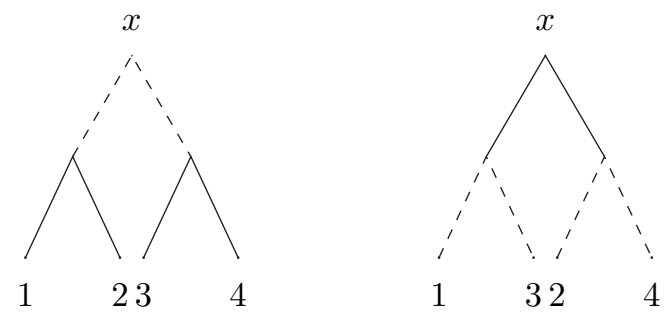

Conversely, $Y$ is called an ascendant of $Z$ and can be obtained after a finite number of simple contractions. Note that this implies that if either of the sets $Y$ or $Z$ is admissible, then so is the other. In fact, the set of admissible subsets of $U_{r}(\Sigma)$ is a poset with respect to the partial order $\leq$. This poset is denoted by $\mathfrak{A}_{r}(\Sigma)$.

It is easy to prove that any admissible subset is a basis of $U_{r}(\Sigma)$ (see [12], Lemma 2.5).

Remark 2.6. Let $\Sigma$ be valid and assume that we have $s$ colours of arities $\left\{n_{1}, \ldots, n_{s}\right\}$. Let $r$ be a positive integer. Observe that the cardinality of any admissible subset of $U_{r}(\Sigma)$ must be of the form $m \equiv r \bmod d$ for

$$
d:=\operatorname{gcd}\left\{n_{i}-1 \mid i=1, \ldots, s\right\} .
$$

Moreover, for any $m \equiv r \bmod d$, there is some admissible subset of cardinality $m$. And as admissible subsets are bases, we get $U_{r}(\Sigma)=U_{m}(\Sigma)$.

Definition 2.7. Let $B, C$ be admissible subsets of $U_{r}(\Sigma)$. We say that $T$ is the unique least upper bound of $B$ and $C$ if $B \leq T, C \leq T$ and for all admissible sets $S$ such that $B \leq S$ and $C \leq S$ we have $T \leq S$.

We say, by abusing notation a little, that $U_{r}(\Sigma)$ is bounded if for all admissible subsets $B, C$ such that there is some admissible $A$ with $A \leq B, C$ there is a unique least upper bound of $B$ and $C$.

One can also define greatest lower bounds, but this places a stronger restriction on the algebra, see [12]. Moreover, note that a priori we require the existence of an upper bound only when our sets have a lower bound $A$, but this turns out to be not too restrictive:

Lemma 2.8. Let $U_{r}(\Sigma)$ be valid and bounded. Then any two admissible subsets have some (possibly not unique) common upper bound.

Proof. Use the same proof as in [12], Proposition 3.4.

Example 2.9. The Brin algebras defined in Example 2.4 are valid and bounded. The existence of a unique least upper bound for $n=2$ is shown in [12], Lemma 3.2. The general case is analogous. 
Example 2.10. Let $P \subseteq \mathbb{Q}>0$ be a finitely generated multiplicative group. Then by a result of Brown ([24], Proposition 1.1), $P$ has a basis of the form $\left\{n_{1}, \ldots, n_{s}\right\}$ with all $n_{i} \geq 0(i=1, \ldots, s)$. Now consider $\Omega$-algebras on $s$ colours of arities $\left\{n_{1}, \ldots, n_{s}\right\}$ and let $\Sigma=\Sigma_{1} \cup \Sigma_{2}$ with $\Sigma_{2}$ the set of identities given by the following orderpreserving identification:

$$
\begin{aligned}
& \left\{\alpha_{i}^{1} \alpha_{j}^{1}, \ldots, \alpha_{i}^{1} \alpha_{j}^{n_{j}}, \alpha_{i}^{2} \alpha_{j}^{1}, \ldots, \alpha_{i}^{2} \alpha_{j}^{n_{j}}, \ldots, \alpha_{i}^{n_{i}} \alpha_{j}^{1}, \ldots, \alpha_{i}^{n_{i}} \alpha_{j}^{n_{j}}\right\} \\
& \quad=\left\{\alpha_{j}^{1} \alpha_{i}^{1}, \ldots, \alpha_{j}^{1} \alpha_{i}^{n_{i}}, \alpha_{j}^{2} \alpha_{i}^{1}, \ldots, \alpha_{j}^{2} \alpha_{i}^{n_{i}}, \ldots, \alpha_{j}^{n_{j}} \alpha_{i}^{1}, \ldots, \alpha_{j}^{n_{j}} \alpha_{i}^{n_{i}}\right\} .
\end{aligned}
$$

Here $i \neq j$ and $i, j \in\{1, \ldots, s\}$.

The Cantor algebras $U_{r}(\Sigma)$ thus obtained will be called Brown-Stein algebras.

Note that, as $\left\{n_{1}, \ldots, n_{s}\right\}$ is a basis for $P$, the $n_{i}$ are all distinct. Hence, when visualising the identities in $\Sigma_{2}$ for the Brown-Stein algebra, it suffices to only use one colour, as the arity of an expansion already determines the colour. In the following example let $r=1, s=2, n_{1}=2$ and $n_{2}=3$.

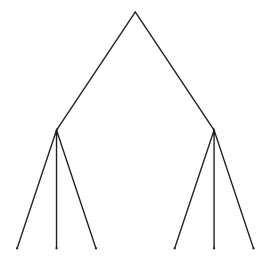

$\begin{array}{llllll}1 & 2 & 3 & 4 & 5 & 6\end{array}$

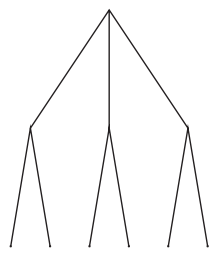

$\begin{array}{llllll}1 & 2 & 3 & 4 & 5 & 6\end{array}$

\section{Lemma 2.11. The Brown-Stein algebras are valid and bounded.}

Proof. This is Proposition 1.2 (due to K. Brown) in [24].

In fact, in [24] Lemma 2.8 is proven directly, i.e., any two admissible subsets have some common upper bound.

We can now define the generalised Higman-Thompson groups. Recall that in a valid Cantor algebra $U_{r}(\Sigma)$ admissible subsets are bases.

Definition 2.12. Let $U_{r}(\Sigma)$ be a valid Cantor algebra. We define $G_{r}(\Sigma)$ to be the group of those $\Omega$-algebra automorphisms of $U_{r}(\Sigma)$ which are induced by a map $V \rightarrow W$, where $V$ and $W$ are admissible subsets of the same cardinality.

Example 2.13. If $U_{r}(\Sigma)$ is a Higman algebra as in Example 2.3, we retrieve the original Higman-Thompson-groups $G_{n, r}$. Let $U_{r}(\Sigma)$ be a Brin algebra on $s$ colours of arity 2 as in Example 2.4. Then the groups constructed are Brin's ([4]) generalisations $s V$ of Thompson's group $V=G_{2,1}$. The description of $s V$ as automorphism groups of a Cantor algebra can be found in [12]. Finally, the groups $G_{r}(\Sigma)$ when $U_{r}(\Sigma)$ is a Brown-Stein algebra as in Example 2.10 were considered in [24]. 
Remark 2.14. It is conceivable that in fact $G_{r}(\Sigma)$ equals the full group of $\Omega$-algebra automorphisms of $U_{r}(\Sigma)$. This would follow if one could prove that any finite basis of $U_{r}(\Sigma)$ is an admissible subset (this is the case for the Higman algebra, see [10] Corollary 1).

We go back to the case of an arbitrary valid Cantor algebra $U_{r}(\Sigma)$ and assume that the set $X$ is ordered. It can be seen that this order is inherited by certain subsets of $W_{\Omega}(X)$ including all admissible subsets, see for example [5] or [10]. If the relations in $\Sigma_{2}$ preserve that ordering, in the sense that the bijection $\phi$ in Definition 2.1 does, then we also have an inherited order on the admissible subsets of $U_{r}(\Sigma)$. We shall call this the induced ordering.

Definition 2.15. Suppose that we have a Cantor algebra $U_{r}(\Sigma)$ where $\Sigma$ preserves the induced ordering. We may define subgroups $F_{r}(\Sigma)$ and $T_{r}(\Sigma)$ of $G_{r}(\Sigma)$ as follows. We let $F_{r}(\Sigma)$ be the group of order-preserving automorphisms between ordered admissible subsets of the same cardinality and $T_{r}(\Sigma)$ the group of cyclic order-preserving automorphisms between ordered admissible subsets of the same cardinality.

Example 2.16. For $U_{r}(\Sigma)$ a Higman algebra of Example 2.3 the definition above yields the groups $F_{n, r}$ and $T_{n, r}$ as in [5]. Recall that Thompson's groups are $F=F_{2,1}$ and $T=T_{2,1}$.

Let $U_{r}(\Sigma)$ be a Brown-Stein algebra as in Example 2.10. In this case, $\Sigma$ is orderpreserving, so we may define the groups $F_{r}(\Sigma)$ and $T_{r}(\Sigma)$, which are considered in [24].

Since $\Sigma_{2}$ in the definition of the Brin algebra of Example 2.4 is not orderpreserving, there is no obvious way to define the groups $F_{r}(\Sigma)$ or $T_{r}(\Sigma)$ for this algebra.

Remark 2.17. Note that if definable, the groups $F_{r}(\Sigma)$ are torsion-free. In both cases mentioned in Example 2.16 the resulting groups $F_{r}(\Sigma)$ are known to be of type $\mathrm{FP}_{\infty}$ and finitely presented ([5], [24]).

Since for torsion-free groups ordinary and Bredon cohomological finiteness conditions are identical we will not consider these groups further.

\section{A model for $\underline{E} G$ for generalised Thompson groups}

From now on we fix a valid $\Sigma$ and a finite positive integer $r$. Also assume that the Cantor algebra $U_{r}(\Sigma)$ is bounded. In this section we give a quite elementary proof of the following result.

Theorem 3.1. The geometric realisation of the poset of admissible subsets is a model for $\underline{E} G_{r}(\Sigma)$. 
We fix an admissible subset $X \subseteq U_{r}(\Sigma)$ of cardinality $r$.

Lemma 3.2. For any finite $Q \leq G_{r}(\Sigma)$ there exists some admissible subset $Z$ such that $Z Q=Z$. Moreover we may assume $X \leq Z$.

Proof. For every $q \in Q$ choose a common upper bound $T_{q}$ of $X$ and $X q$. Then put $Z_{q}:=T_{q} q^{-1}$ and let $Y$ be an upper bound of

$$
\left\{Z_{q} \mid q \in Q\right\} .
$$

Note that $X \leq Z_{1}=T_{1}$ and for any $q \in Q$,

$$
X \leq T_{q}=Z_{q} q \leq Y q .
$$

Therefore we may choose $Z$ the least upper bound of $\{Y q \mid q \in Q\}$. By definition of unique least upper bound we get $Z Q=Z$.

Proposition 3.3. Any two elements in $\mathfrak{A}_{r}(\Sigma)^{Q}$ have an upper bound in $\mathfrak{A}_{r}(\Sigma)^{Q}$.

Proof. Let $Y, Z \in \mathfrak{A}_{r}(\Sigma)^{Q}$. We begin by showing that there are admissible sets $Y_{1}, Z_{1} \in \mathfrak{A}_{r}(\Sigma)^{Q}$ such that $Y_{1}$ is an upper bound of $X$ and $Y$ and $Z_{1}$ is an upper bound of $X$ and $Z$. It suffices to prove the existence of $Y_{1}$. Take an upper bound $Y_{2} \in \mathfrak{A}_{r}(\Sigma)$ of $X$ and $Y$ and consider

$$
\left\{Y_{2} q^{-1} \mid q \in Q\right\} \text {. }
$$

Let $Y_{3} \in \mathfrak{A}_{r}(\Sigma)$ be an upper bound of this set. Then, for any $q \in Q$,

$$
Y_{2} \leq Y_{3} q .
$$

Therefore $X \leq Y_{3} q$. This implies that we may choose $Y_{1}$ to be the least upper bound of

$$
\left\{Y_{3} q \mid q \in Q\right\} \text {. }
$$

Clearly, $Y, X \leq Y_{1}$. Again, the definition of least upper bound implies that $Y_{1} \in$ $\mathfrak{A}_{r}(\Sigma)^{Q}$.

Now let $T$ be the least upper bound of $Y_{1}$ and $Z_{1}$. Then for any $q \in Q$,

$$
Y_{1}=Y_{1} q \leq T q, \quad Z_{1}=Z_{1} q \leq T q,
$$

so we get $T \in \mathfrak{A}_{r}(\Sigma)^{Q}$.

Proof of Theorem 3.1. Lemmas 3.2 and 3.3 imply that for any finite subgroup $Q \leq G_{r}(\Sigma)$ the poset $\mathfrak{A}_{r}(\Sigma)^{Q}$ is non-empty and directed, thus $\left|\mathfrak{A}_{r}(\Sigma)\right|^{Q}=$ $\left|\mathfrak{A}_{r}(\Sigma)^{Q}\right| \simeq *$. Moreover for any $V \in \mathfrak{A}_{r}(\Sigma)$,

$$
\operatorname{Stab}_{G_{r}(\Sigma)}(V)=\left\{g \in G_{r}(\Sigma) \mid V g=V\right\}
$$

is contained in the group of permutations of the finite set $V$, thus it is finite. This implies that for any $H \leq G_{r}(\Sigma), \mathfrak{A}_{r}(\Sigma)^{H}=\varnothing$ unless $H$ is finite. 
This model is not of finite type, but there is a filtration of $\left|\mathfrak{U}_{r}(\Sigma)^{Q}\right|$ by finite type subcomplexes, exactly as in the construction in [5], Theorem 4.17:

Proposition 3.4. For any finite $Q \leq G_{r}(\Sigma)$ there is a filtration of $\left|\mathfrak{U}_{r}(\Sigma)^{Q}\right|$,

$$
\cdots \subset\left|\mathfrak{U}_{r}(\Sigma)^{Q}\right|_{h-1} \subset\left|\mathfrak{A}_{r}(\Sigma)^{Q}\right|_{h} \subset\left|\mathfrak{U}_{r}(\Sigma)^{Q}\right|_{h+1} \subset \cdots,
$$

such that each $\left|\mathfrak{U}_{r}(\Sigma)^{Q}\right|_{h} / C_{G_{r}(\Sigma)}(Q)$ is finite.

Proof. Let

$$
\left|\mathfrak{U}_{r}(\Sigma)^{Q}\right|_{h}:=\left\{Y \in \mathfrak{U}_{r}(\Sigma)^{Q}|| Y \mid \leq h\right\} .
$$

Consider $Y, Z \in \mathfrak{U}_{r}(\Sigma)^{Q}$ with $|Y|=|Z|$ and isomorphic as $Q$-sets. This means that there is a $Q$-bijection

$$
\sigma: Y \rightarrow Z
$$

Let $g \in G_{r}(\Sigma)$ be the element given by $y g=y \sigma$ for each $y \in Y$. Then for any $q \in Q,(y q) g=(y q) \sigma=y \sigma q=y g q$. This means that the commutator $[g, q]$ acts as the identity on the admissible set $Y$ and therefore $[g, q]=1$. Hence $g \in C_{G_{r}(\Sigma)}(Q)$. As for any $m \leq h$ there are finitely many possible $Q$-sets of cardinality $m$, the result follows.

Remark 3.5. Provided that $\Sigma$ is order-preserving, Theorem 3.1 and Proposition 3.4 can be restated replacing $G_{r}(\Sigma)$ with $T_{r}(\Sigma)$.

Remark 3.6. The filtration of Proposition 3.4 is used by Brown [5], Theorem 4.17, with $Q=1$, to show that the Higman-Thompson groups $G_{n, r}, T_{n, r}$ and $F_{n, r}$ are of type $\mathrm{FP}_{\infty}$. The approach used by Brown is as follows: Fix an admissible subset $Y$. Show that if $\left|\mathfrak{A}_{n, r}\right|_{<Y}$ denotes the set of admissible subsets which are contractions of $Y$, then the connectivity of $\left|\mathfrak{A}_{n, r}\right|_{<Y}$ grows with the cardinality of $Y$. Then, show that this implies that the connectivity of the pair $\left(\left|\mathfrak{U}_{r, n}\right|_{h+1},\left|\mathfrak{A}_{r, n}\right|_{h}\right)$ tends to $\infty$, which in turn yields that $G_{n, r}, T_{n, r}$ and $F_{n, r}$ are all of type $\mathrm{FP}_{\infty}$. Key to this approach is understanding the complex $\left|\mathfrak{A}_{n, r}\right|_{<Y}$. In the case of the Higman algebra, Brown shows ([5], Lemmas 4.18, 4.19) that any two simple contractions $Y_{1}, Y_{2}$ of $Y$ have a common lower bound if and only if the contracted vertices are disjoint, which allows him to show that $\left|\mathfrak{U}_{n, r}\right|_{<Y}$ is homotopy equivalent to a much simpler complex. However, this is no longer true if we work with a more general Cantor algebra $U_{r}(\Sigma)$ : Consider for example a Brown-Stein algebra as in Example 2.10 with arities 2 and 3. Let $Y$ be any admissible set with 6 elements labeled 1, 2, 3, 4, 5 and 6. Let $Y_{1}$ be the simple contraction of arity 2 of the elements 3 and 4 and $Y_{2}$ the simple contraction of arity 3 of the elements 1,2 and 3 . Then the sets of contracted vertices are not disjoint, however there is a common lower bound $Z \leq Y_{1}, Y_{2}$ as the picture before Lemma 2.11 shows. Stein used a different method to the one described here to prove that the groups of [24] are of type $\mathrm{FP}_{\infty}$. 
Similar problems were encountered when Kochloukova and the authors considered Brin's groups [12]. In general, the same difficulty applies to the groups $G_{r}(\Sigma)$, as well as to $T_{r}(\Sigma), F_{r}(\Sigma)$ where definable. It is conceivable, however, that Brown's approach can be applied more generally using an analogue of Brown's connectivity result, see for example [12] where it is used to show that Brin's groups for $r=1$, $s=2,3$ are of type $\mathrm{FP}_{\infty}$.

\section{Centralisers and conjugacy classes of finite subgroups for $G_{r}(\Sigma)$ and $T_{r}(\Sigma)$}

Let $Q \leq G_{r}(\Sigma)$ be a finite subgroup. In this section we give a more detailed analysis of the poset $\mathfrak{A}_{r}(\Sigma)^{Q}$ to describe $C_{G_{r}(\Sigma)}(Q)$ and the number of conjugacy classes of subgroups isomorphic to $Q$. In case $T_{r}(\Sigma)$ is defined, we also derive the corresponding results. This will be used later when we prove our main result on the cohomological finiteness properties of these groups.

Let $\left\{w_{1}, \ldots, w_{t}\right\}$ be the set of lengths of all the possible transitive permutation representations of $Q$. Any $Y \in \mathfrak{A}_{r}(\Sigma)^{Q}$ is a finite $Q$-set so it is determined by its decomposition into transitive $Q$-sets. If we take one of those sets and apply the operation $\alpha_{i}$ for a fixed colour $i$ to each of its elements, we obtain a new admissible subset which is also fixed by $Q$. We say that this is a simple $Q$-expansion of $Y$. More explicitly, the admissible set obtained from $Y$ is:

$$
Y \backslash\{y q \mid q \in Q\} \cup\left\{y q \alpha_{i}^{j} \mid q \in Q, 1 \leq j \leq n_{i}\right\}
$$

for a certain $y \in Y$. We also use the term $Q$-expansion to refer to a chain of simple $Q$-expansions.

Conversely, if we choose $n_{i}$ different orbits of the same type (i.e., corresponding to the same permutation representation) in $Y$, then we may contract them to a single orbit (of the same type). We call this a simple $Q$-contraction. Simple $Q$-contractions are more complicated to handle than simple $Q$-expansions: we may contract an element of the first of the orbits with any of the elements on the others. Hence, even if the orbits to be contracted are determined, there are many possibilities to perform the explicit contraction. Note that the admissible subsets obtained this way will lie in $\mathfrak{A}_{r}(\Sigma)^{Q}$.

Large parts of the next three results can be found in [10], Section 6 . We shall, for the reader's convenience, recall the arguments.

Lemma 4.1. Let $Y, Z \in \mathfrak{A}_{r}(\Sigma)^{Q}$ with $Y<Z$ and assume there is no admissible subset $C \in \mathfrak{A}_{r}(\Sigma)^{Q}$ with $Y \leq C \leq Z$. Then $Z$ is a simple $Q$-expansion of $Y$. Hence $Y$ is a simple $Q$-contraction of $Z$.

Proof. We may choose a chain of simple expansions

$$
Y<Y_{1}<\cdots<Y_{r}<Z \text {. }
$$


Let $w \in Y$ be the vertex expanded in the first simple expansion $Y<Y_{1}$ and $W \subseteq Y$ be the $Q$-orbit with $w \in W$. Assume also that this first expansion corresponds to the colour $i$. Then as $Z$ contains certain descendants of $\left\{w \alpha_{i}\right\}$ and it is $Q$-invariant it must also contain the analogous descendants of $\left\{u \alpha_{i} \mid u \in W\right\}$. Therefore if $C$ denotes the simple $Q$-expansion consisting of expanding $W$ by $\alpha_{i}$, then $Y<C \leq Z$. As $C \in \mathfrak{U}_{r}(\Sigma)^{Q}$, we deduce by the hypothesis that $C=Z$.

Proposition 4.2. For any finite subgroup $Q \leq G_{r}(\Sigma)$, there is a uniquely determined set of integers $\pi(Q):=\left\{r_{1}, \ldots, r_{t}\right\}$ with $0 \leq r_{j} \leq d$ and

$$
\sum_{j=1}^{t} r_{j} w_{j} \equiv r \bmod d
$$

such that there is an admissible subset $Y \in \mathfrak{U}_{r}(\Sigma)^{Q}$ with $|Y|=\sum_{j=1}^{t} r_{j} w_{j}$.

Moreover, any other element in $\mathfrak{U}_{r}(\Sigma)^{Q}$ can be obtained from $Y$ by a finite sequence of simple $Q$-expansions or $Q$-contractions.

Proof. First, note that by Lemma 3.2, $\mathfrak{A}_{r}(\Sigma)^{Q} \neq \varnothing$. Now choose some $Z \in$ $\mathfrak{U}_{r}(\Sigma)^{Q}$ and decompose it as a disjoint union of transitive $Q$-sets. Let $k_{j}$ be the number of transitive sets in this decomposition which are of type $j$, i.e., which correspond to the same permutation representation. Observe that whenever we apply simple $Q$-contractions or $Q$-expansions to $Z$, if the set thus obtained has $m_{j}$ transitive $Q$-sets of type $j$, then $m_{j} \equiv k_{j} \bmod d$. Note also that

$$
|Z|=\sum_{j=1}^{t} k_{j} w_{j} \equiv r \bmod d .
$$

Let

$$
r_{j}= \begin{cases}0 & \text { if } k_{j}=0 \\ d & \text { if } 0 \neq k_{j} \equiv 0 \bmod d \\ l & \text { with } 0<l<d \text { and } l \equiv k_{j} \bmod d \text { otherwise }\end{cases}
$$

By successively performing simple $Q$-contractions or $Q$-expansions of $Z$ we may get an admissible set $Y$ such that the number of transitive $Q$-sets of type $j$ in $Y$ is exactly $r_{j}$. Observe that the $r_{j}$ are uniquely determined, whereas $Y$ is not. Finally, Proposition 3.3 implies that for any other $C \in \mathfrak{A}_{r}(\Sigma)^{Q}$ there is an upper bound, say $D$, of $Y$ and $C$ with $D \in \mathfrak{U}_{r}(\Sigma)^{Q}$ which means that

$$
Y \leq D \geq C
$$

By Lemma 4.1 we may choose chains

$$
Y=D_{0}<D_{1}<\cdots<D_{l_{1}}=D=C_{0}>C_{1}>\cdots>C_{l_{2}}=C
$$

such that each step consists of a simple $Q$-expansion/ $Q$-contraction, and we are done. 
Theorem 4.3. Let $Q_{1}, Q_{2} \leq G_{r}(\Sigma)$ be finite subgroups with $Q_{1} \cong Q_{2}$. Then $Q_{1}$ and $Q_{2}$ are conjugate in $G_{r}(\Sigma)$ if and only if $\pi\left(Q_{1}\right)=\pi\left(Q_{2}\right)$.

In particular, there are only finitely many conjugacy classes of subgroups isomorphic to $Q_{1}$.

Proof. Fix an isomorphism $\phi: Q_{1} \rightarrow Q_{2}$. Assume first that $\pi\left(Q_{1}\right)=\pi\left(Q_{2}\right)$. Then there are admissible subsets $V_{1}, V_{2}$ with $V_{i} \in \mathfrak{A}_{r}(\Sigma){ }^{Q_{i}}$ having the same number of elements and moreover the same structure as $Q_{i}$-sets, which means that there is a bijection between them which we denote by $g$ such that for any $q \in Q_{1}$ and $v \in V_{1}$, $(v q) g=v g q^{\phi}$. This yields an element $g \in G_{r}(\Sigma)$ with $g^{-1} q g=q^{\phi}$.

Conversely, assume $Q_{2}=g^{-1} Q_{1} g$ with $g \in G_{r}(\Sigma)$. Then for any $V_{1} \in$ $\mathfrak{A}_{r}(\Sigma)^{Q_{1}}, V_{1} g \in \mathfrak{A}_{r}(\Sigma)^{Q_{2}}$. Moreover, $g$ induces an isomorphism as $Q_{i}$-sets so the orbit structure of the minimal elements of $\mathfrak{A}_{r}(\Sigma)^{Q_{1}}$ and $\mathfrak{A}_{r}(\Sigma)^{Q_{1}}$ has to be the same.

Theorem 4.4. Let $Q \leq G_{r}(\Sigma)$ be a finite subgroup and $\pi(Q)=\left\{r_{1}, \ldots, r_{t}\right\}$ as in Proposition 4.2. Then $C_{G_{r}(\Sigma)}(Q) \cong H_{r_{1}} \times \cdots \times H_{r_{t}}$ where each of the $H_{r_{i}}$ fits into the following split group extension

$$
K_{i} \succ H_{r_{i}} \rightarrow G_{r_{i}}(\Sigma)
$$

with $K_{i}$ locally finite.

Proof. Choose an admissible $Y \in \mathfrak{A}_{r}(\Sigma)^{Q}$ as in Proposition 4.2. We begin by proving the result in the special case when there are exactly $l Q$-orbits all of the same type in the $Q$-set $Y$. In other words, we assume that in Proposition 4.2 for some $k, l:=r_{k}$ and all the others $r_{j}=0$. Let $w:=w_{k}$ be the length of those $Q$-orbits, and for each $i=1, \ldots, l$, choose an orbit representative $y_{i}$. We call the subset $\left\{y_{1}, \ldots, y_{l}\right\} \subseteq Y$ thus obtained the set of marked elements. We consider any $Q$-expansion of $Y$ as marked, by marking precisely the descendants of marked elements in $Y$. And we say that a $Q$-contraction is marked if marked elements are contracted only with marked elements and result in the marked elements of the new subset. Note that this implies that elements of the form $y q$ with $q \in Q$ and $y$ marked can only be contracted with elements $y^{\prime} q$ for the same $q \in Q$ and $y^{\prime}$ marked. We now define

$$
\begin{gathered}
\mathcal{M}=\left\{M \mid M \in \mathfrak{A}_{r}(\Sigma)^{Q} \text { admissible and obtained from } Y\right. \text { by marked } \\
Q \text {-expansions and marked } Q \text {-contractions }\} .
\end{gathered}
$$

$\mathcal{M}$ is the set of marked admissible subsets of $\mathfrak{A}_{r}(\Sigma)^{Q}$ and can also be seen as the diagonal subposet:

$$
\mathcal{M} \subseteq \underbrace{\mathfrak{A}_{l}(\Sigma) \times \cdots \times \mathfrak{A}_{l}(\Sigma)}_{w} .
$$


Now fix an admissible subset $X=\left\{x_{1}, \ldots, x_{l}\right\} \in \mathfrak{A}_{l}(\Sigma)$ and fix a bijection $\iota_{X, Y}: x_{i} \mapsto y_{i}$ from $X$ to the marked elements of $Y$. From $X \mapsto Y$ we get a poset map

$$
\iota^{\mathfrak{A}}: \mathfrak{A}_{l}(\Sigma) \rightarrow \mathfrak{A}_{r}(\Sigma)^{Q},
$$

which commutes with expansions and contractions in $\mathfrak{A}_{l}(\Sigma)$, and with $Q$-expansions and marked $Q$-contractions in $\mathfrak{A}_{r}(\Sigma)^{Q}$. This is well defined since $X$ is a basis of the algebra used to construct $\mathfrak{X}_{l}(\Sigma)$. The fact that we only contract marked elements allows us to avoid ambiguities. Observe that $\operatorname{im}\left(\iota^{\mathfrak{T}}\right)=\mathcal{M}$. Moreover, whenever $\iota^{\mathfrak{2}}\left(X_{1}\right)=Y_{1}$, there is a well-defined bijection $\iota_{X_{1}, Y_{1}}$ between $X_{1}$ and the set of marked elements in $Y_{1}$. For convenience we let $\iota_{X, Y}$ act on the right. We use this to define a group homomorphism

$$
\iota: G_{l}(\Sigma) \rightarrow C_{G_{r}(\Sigma)}(Q),
$$

as follows: Let $g$ be given by a map $g: X_{1} \rightarrow X_{2}$ for $X_{1}, X_{2} \in \mathfrak{A}_{r}(\Sigma)$ and put $Y_{1}=$ $\iota^{\mathfrak{2}}\left(X_{1}\right), Y_{2}=\iota^{\mathfrak{2}}\left(X_{2}\right)$. Then $\iota(g): Y_{1} \rightarrow Y_{2}$ is the unique map which commutes with the $Q$-action and such that $g \iota_{X_{2}, Y_{2}}=\iota_{X_{1}, Y_{1}} \iota(g)$ (recall that the marked elements are representatives of the $Q$-orbits). Obviously $\iota(g) \in C_{G_{r}(\Sigma)}(Q)$.

Next we define a second poset map

$$
\tau^{\mathfrak{A}}: \mathfrak{A}_{r}(\Sigma)^{Q} \rightarrow \mathfrak{A}_{l}(\Sigma)
$$

such that $\tau^{\mathfrak{A}} l^{\mathfrak{A}}=\operatorname{id}_{\mathfrak{A}_{l}(\Sigma)}$. To do this, put $\tau^{\mathfrak{A}}(Y)=X$, identify all the elements in the $Q$-orbit of each $y_{i}$ with $x_{i}$ and extend using the corresponding operations on both sides. Proposition 4.2 and the fact that $Y$ is admissible, imply that $\mathfrak{A}_{r}(\Sigma)^{Q}$ is also free on $Y$, hence $\tau^{\mathfrak{I}}$ is well defined. In an analogous way as before, there is also an explicit bijection between the $Q$-orbits in any $Y_{1}$ and the elements of $\tau^{\mathfrak{A}}\left(Y_{1}\right)$ which can be used to define a group homomorphism

$$
\tau: C_{G_{r}(\Sigma)}(Q) \rightarrow G_{l}(\Sigma) .
$$

Observe that whenever $g \in C_{G_{r}(\Sigma)}(Q)$ and $Y_{1} \in \mathfrak{A}_{r}(\Sigma)^{Q}$, then $Y_{1} g \in \mathfrak{A}_{r}(\Sigma)^{Q}$.

In particular, $\tau \iota=\operatorname{id}_{G_{l}(\Sigma)}$, giving us the desired split group extension. We now proceed to describe $K:=\operatorname{ker} \tau$. To begin we observe that $K$ consists precisely of those $h \in C_{G_{r}(\Sigma)}(Q)$ such that for any $A \in \mathfrak{A}_{r}(\Sigma)^{Q}, \tau^{\mathfrak{A}}(A h)=\tau^{\mathfrak{A}}(A)$ and $h$ fixes the $Q$-orbits of $A$ setwise.

We claim that for any $h \in K$ there is some $Q$-expansion of $Y, Z \in \mathfrak{A}_{r}(\Sigma)^{Q}$ with $Z h=Z$. To see this, using Proposition 3.3, take $Z \in \mathfrak{A}_{r}(\Sigma)^{Q}$ to be an upper bound of $Y, Y h^{-1}$. Then Lemma 4.1 implies that $Z$ and $Z h$ are both $Q$-expansions of $Y$ and therefore they are marked. Thus $Z, Z h \in \mathcal{M}=\operatorname{im} \iota^{\mathfrak{T}}$. As $h$ lies in $K$, we have $\tau^{\mathfrak{A}}(Z)=\tau^{\mathfrak{A}}(Z h)$. So the fact that $\tau^{\mathfrak{A}}$ is injective when restricted to $\operatorname{im} \iota^{\mathfrak{A}}$ implies the claim. In particular, $K$ is the union of its subgroups of the form

$$
K_{Z}:=\left\{h \in C_{G_{r}(\Sigma)}(Q) \mid Z h=Z, h \text { fixes the } Q \text {-orbits setwise }\right\},
$$


where $Z$ is a $Q$-expansion of $Y$. As each $K_{Z}$ is finite, using Proposition 3.3 we see that $K$ is locally finite, thus proving the special case.

To finish our proof, we now prove the general case when $Y$ has $Q$ orbits of different types. Let $\pi(Q)=\left\{r_{1}, \ldots, r_{t}\right\}$ and $w_{1}, \ldots, w_{t}$ be as in Proposition 4.2. Let $Y=\bigcup_{i=1}^{t} Y_{i}$ with $Y_{i}$ the union of the $r_{i} Q$-orbits of type $i$ in $Y$. Then $Q$ acts on each $Y_{i}$. Note that a single action might not be faithful, but the intersection of the kernels must be trivial. Also note that in $\mathfrak{A}_{r}(\Sigma) Q, Q$-contractions can not mix elements belonging to orbits of different type. This implies that we have a direct product of posets

$$
\mathfrak{A}_{r}(\Sigma)^{Q} \cong \mathfrak{A}_{w_{1} r_{1}}(\Sigma)^{Q} \times \cdots \times \mathfrak{A}_{w_{t} r_{t}}(\Sigma)^{Q},
$$

where we let the group $Q$ act on each poset $\mathfrak{A}_{w_{i} r_{i}}(\Sigma)$ using its action on $Y_{i}$ and extending via extensions and contractions. This action yields also a group homomorphism $\phi_{i}: Q \rightarrow G_{w_{i} r_{i}}(\Sigma)$. The direct product of posets above implies that $C_{G_{r}(\Sigma)}(Q)$ decomposes as the direct product of the centralisers of $\phi_{i}(Q) \leq G_{w_{i} r_{i}}(\Sigma)$. For each of these we can apply the case of a single type of orbit, and we are done.

Remark 4.5. In an analogous way, one can prove that there is also a group epimorphism

$$
N_{G_{r}(\Sigma)}(Q) \rightarrow G_{r_{1}}(\Sigma) \times \cdots \times G_{r_{t}}(\Sigma)
$$

with locally finite kernel.

Remark 4.6. In [3], there is a description of centralisers of elements $g$ in the Higman groups $G_{n, 1}$ associated to $U_{r}(\Sigma)=V_{n, 1}$. Whenever $Q=\langle g\rangle$ has finite order in those groups, this coincides with ours.

Remark 4.7. With little more effort we can give a description of the kernel $K$ appearing in the single type of orbit case in the proof of Theorem 4.4: Let $S_{w}$ be the symmetric group of degree $w$ and choose a permutation representation $\phi: Q \rightarrow S_{w}$ associated to the $Q$ action on the orbits of $Y$. Denote $L:=C_{S_{w}}(\phi(Q))$. Then if $Z$ is a $Q$-expansion of $Y$ with $|Z|=m w$, we have the following isomorphism:

$$
\left\{h \in C_{G_{r}(\Sigma)}(Q) \mid Z h=Z, h \text { fixes the } Q \text {-orbits setwise }\right\} \cong L^{m}:=\underbrace{L \times \cdots \times L}_{m} .
$$

Consider now a simple $Q$-expansion $Z \leq Z_{1}$ with $\left|Z_{1}\right|=m_{1} w$, consisting of applying a descending operation of arity $n_{j}$ to a vertex $z_{0}$. We get a group homomorphism $L^{m} \rightarrow L^{m_{1}}$ given by mapping the copy of $L$ corresponding to $z_{0}$ to the product of copies of $L$ corresponding to the descendants of $z_{0}$ via the diagonal map $L \rightarrow L^{n_{j}}$, and leaving the remaining factors intact. This gives, in an obvious way, a direct system of groups and hence $K$ is the directed limit of the system thus obtained. 
We shall now consider the groups $T_{r}(\Sigma)$ whenever they are defined, i.e., whenever $\Sigma$ preserves the induced order in the admissible subsets of our Cantor algebra $U_{r}(\Sigma)$. In this case, centralisers of finite subgroups have an easier structure.

A first observation is that any finite subgroup $Q \leq T_{r}(\Sigma)$ is cyclic. Moreover following the argument of Proposition 4.2 we see that by writing the transitive permutation representations of $Q$ to have the faithful representation first, i.e., $w_{1}=|Q|$, we obtain $\pi(Q)=\left\{r_{1}, 0, \ldots, 0\right)$ and $r_{1} w_{1} \equiv r \bmod d$. To see this, take for example $Y \in \mathfrak{U}_{r}(\Sigma)^{Q}$ the admissible subset obtained following the argument of Proposition 4.2 and assume that certain $g \in Q$ fixes some $y_{0} \in Y$. The condition that $g$ preserves cyclically the order, implies that $g$ fixes $Y$ pointwise, thus $g=1$. As a consequence, $|Y|=r_{1} w_{1}$.

Theorem 4.8. Let $U_{r}(\Sigma)$ be a Cantor algebra with order-preserving $\Sigma$ and $Q \leq$ $T_{r}(\Sigma)$ a finite subgroup. Then there is only one conjugacy class of finite subgroups of order $|Q|$ and for a certain $0<l \leq d$ depending on $Q$ there is a central extension

$$
Q \succ C_{T_{r}(\Sigma)}(Q) \rightarrow T_{l}(\Sigma)
$$

Proof. For the first assertion observe that any two cyclic groups of the same order are isomorphic and they only have one faithful permutation representation. Hence it suffices to choose a cyclic order-preserving $h$ between the corresponding admissible subsets $Y$. Note that they have the same cardinality.

For the second assertion, embed $T_{r}(\Sigma)$ in $G_{r}(\Sigma)$ and let $\iota, \tau$ be the group homomorphisms of Theorem 4.4, we use the same notation as there. The result will follow once we check that $\tau\left(C_{T_{r}(\Sigma)}(Q)\right)=T_{l}(\Sigma)$ and that $Q=\operatorname{ker} \tau \cap T_{r}(\Sigma)$.

Note first that we may choose the map $\iota_{X, Y}$ to be order-preserving. The fact that the action of $Q$ cyclically preserves the order on $Y$, implies that we may assume that if for any basis $Y_{1}$ the marked elements are $\left\{y_{1}, \ldots, y_{m}\right\}$, then the elements of $Y_{1}$ are ordered as

$$
y_{1}<\cdots<y_{m}<y_{1} q<\cdots<y_{m} q<\cdots<y_{1} q^{i}<\ldots y_{m} q^{i}<\cdots
$$

for certain (fixed) $q$ generating $Q$ (note that the marked elements of elements of $Y$ can be chosen so that $Y$ is ordered this way). If $g \in C_{T_{r}(\Sigma)}(Q)$ represents a map between two such sets cyclically preserving that ordering, then it is obvious that the corresponding map $\tau(g)$ also does. In fact, write $y_{1}^{\prime}<\cdots<y_{m}^{\prime}<y_{1}^{\prime} q<$ $\cdots<y_{m}^{\prime} q<\cdots<y_{1}^{\prime} q^{i}<\ldots y_{m}^{\prime} q^{i}<\cdots$ for the elements of $Y_{1} g$ and choose the index $j$ such that $y_{j} g=y_{m}^{\prime} q^{a}$. Then if $j<m, y_{j+1} g=y_{1}^{\prime} q^{a+1}$ and if $j=m$, $y_{1} g=y_{1}^{\prime} q^{a}$. Here $0 \leq a \leq|Q|-1$. This implies $\tau\left(C_{T_{r}(\Sigma)}(Q)\right) \subseteq T_{l}(\Sigma)$.

Conversely, take $g \in T_{l}(\Sigma)$. Then $g$ is determined by its action on a pair of ordered admissible subsets $X_{1}: x_{1}<\cdots<x_{m}, X_{2}=X_{1} g: x_{1}^{\prime}<\cdots<x_{m}^{\prime}$. Put $Y_{1}:=\iota^{\mathfrak{A}}\left(X_{1}\right), Y_{2}:=\iota^{\mathfrak{A}}\left(X_{2}\right)$ and denote their elements as before. Now, $\iota(g)$ as defined in Theorem 4.4 does not cyclically preserve the order between $Y_{1}$ and $Y_{2}$. 
Let $j$ be the subindex such that $x_{1} g=x_{j}^{\prime}$. We construct $k \in K=\operatorname{ker} \tau$ as follows:

$$
y_{i}^{\prime} q^{a} k= \begin{cases}y_{i}^{\prime} q^{a+1} & \text { for } 1 \leq i<j, \\ y_{i}^{\prime} q^{a} & \text { for } j \leq i \leq m,\end{cases}
$$

where, as before, $0 \leq a \leq|Q|-1$. A routine check shows that this is well defined and $\iota(g) k: Y_{1} \rightarrow Y_{2}$ cyclically preserves the order between $Y_{1}$ and $Y_{2}$; in other words, $\iota(g) k \in T_{r}(\Sigma)$. From this we deduce that $\tau: C_{T_{r}(Q)}(Q) \rightarrow T_{l}(\Sigma)$ is an epimorphism.

Finally, recall that by the proof of Theorem 4.4 and Remark 4.7, $\operatorname{ker} \tau$ is the union of its subgroups of the form

$$
K_{Z}=\left\{h \in C_{G_{r}(\Sigma)}(Q) \mid Z h=Z \text { and } h \text { fixes the } Q \text {-orbits setwise }\right\} \cong L^{m}
$$

for each $Q$-expansion $Z$ of $Y$. Moreover, as $Q$ is transitive and regular, $L=$ $C_{S_{w_{1}}}(\phi(Q)) \cong Q$. The observation before this theorem implies that the finite group $K_{Z} \cap T_{r}(\Sigma)$ must in fact act in the same way as $Q$ acts on $Z$. Hence

$$
K_{Z} \cap T_{r}(\Sigma)=Q .
$$

Note that under the isomorphism $K_{Z} \cong Q^{m}$, this corresponds to the diagonal subgroup of $Q^{m}$. That the extension is central now follows immediately.

Remark 4.9. For the Higman algebra $U_{r}(\Sigma)=V_{2,1}$ and $T_{r}(\Sigma)=T$, the original Thompson group $T$, this reproves [19], Theorem 7.1.5.

\section{Finiteness conditions in Bredon cohomology}

In this section we collect all necessary background on Bredon cohomological finiteness conditions and also prove an analogue to Bieri's criterion for $\mathrm{FP}_{n}$.

Let $\mathcal{X}$ denote a family of subgroups of a given group $G$. In Bredon cohomology, the group $G$ is replaced by the orbit category $\mathcal{O}_{\chi} G$. The category $\mathcal{O}_{\chi} G$ has as objects the transitive $G$-sets with stabilisers in $\mathcal{X}$. Morphisms in $\mathcal{O}_{X} G$ are $G$-maps between those $G$-sets. Modules over the orbit category, called $\mathcal{O}_{x} G$-modules, are contravariant functors from the orbit category to the category of abelian groups. Exactness is defined pointwise: a sequence

$$
A \rightarrow B \rightarrow C
$$

of $\mathcal{O}_{x} G$-modules is exact at $B$ if and only if

$$
A(\Delta) \rightarrow B(\Delta) \rightarrow C(\Delta)
$$

is exact at $B(\Delta)$ for every transitive $G$-set $\Delta$. 
The category $\mathcal{O}_{x} G$ - $\mathfrak{M D D}_{\mathrm{D}}$ of $\mathcal{O}_{x} G$-modules has enough projectives, which are constructed as follows: For any $G$-sets $\Delta$ and $\Omega$, denote by $[\Delta, \Omega]$ the set of $G$-maps from $\Delta$ to $\Omega$. Let $\mathbb{Z}[\Delta, \Omega]$ be the free abelian group on $[\Delta, \Omega]$. One now obtains an $\mathcal{O}_{X} G$-module $\mathbb{Z}[-, \Omega]$ by fixing $\Omega$ and letting $\Delta$ range over the transitive $G$-sets with stabilisers in $\mathcal{X}$. A Yoneda-type argument, see [21], yields that these modules are free. In particular, the modules $P^{K}(-)=\mathbb{Z}[-, G / K]$ for $K \in \mathcal{X}$ are free and can be viewed as the building blocks for free $\mathcal{O}_{x} G$-modules. Projective modules are now defined analogously to the ordinary case. The trivial $\mathcal{O}_{x} G$-module, denoted by $\mathbb{Z}(-)$, is the constant functor $\mathbb{Z}$ from $\mathcal{O}_{x} G$ to the category of abelian groups.

Bieri [1] gives criteria for a $\mathbb{Z} G$-module to be of type $\mathrm{FP}_{n}$ involving certain Extand Tor-functors commuting with exact colimits and direct products respectively. In this section we prove that those criteria can also be used for Bredon cohomology. The Bredon cohomology functors $\operatorname{Ext}_{\mathcal{X}}^{*}(M,-)$ are defined as derived functors of $\operatorname{Hom}_{\mathfrak{X}}(M,-)$. In particular, let $M(-) \in \mathcal{O}_{X} G-\mathfrak{M D D}_{\mathfrak{D}}$ be a contravariant $\mathcal{O}_{\mathcal{F}} G$ module admitting a projective resolution $P_{*}(-) \rightarrow M(-)$. Then, for each $N(-) \in$ $\mathcal{O}_{x} G-\mathfrak{M o d}$

$$
\operatorname{Ext}_{\mathfrak{X}}^{*}(M, N)=H_{*}\left(\operatorname{Mor}\left(P_{*}, N\right)\right) .
$$

One can also define Bredon homology functors $\operatorname{Tor}_{*}^{\mathfrak{x}}(-, M)$. In particular, by analogy with the contravariant case, one can define covariant $\mathcal{O}_{x} G$-modules, or just comodules for short. The category of covariant $\mathcal{O}_{x} G$-modules, denoted by $\mathfrak{M}_{\mathrm{DD}}$ $\mathcal{O}_{x} G$, behaves just as expected. For example, we have short exact sequences and enough projectives as above. In particular, the building blocks for projective modules in $\mathfrak{M D D}_{\mathcal{X}} \mathcal{O}_{X} G$ are the covariant functors $P_{K}(-)=\mathbb{Z}[G / K,-]$ for subgroups $K \in \mathfrak{X}$. Let $M(-) \in \mathcal{O}_{x} G-M_{\mathfrak{D} D}$ be as before. Then Bredon homology functors are the derived functors of $-\otimes_{\mathfrak{X}} M$, i.e., for any $L(-) \in \mathfrak{M D D}_{\mathfrak{D}} \mathcal{O}_{\mathfrak{X}} G$,

$$
\operatorname{Tor}_{*}^{\mathfrak{X}}(L, M)=H_{*}\left(L \otimes \mathfrak{X} P_{*}\right) .
$$

For details on these definitions including the categorical tensor product and Yoneda-type isomorphism the reader is referred to [22]. In particular, $\operatorname{Tor}_{*}^{\mathfrak{X}}(-, M)$ can be calculated using flat resolutions of $M(-)$.

The category of $\mathcal{O}_{X} G$-modules, as an abelian category, has well-defined colimits and limits and in particular coproducts and products. We say a functor

$$
T: \mathcal{O}_{X} G-\mathfrak{M O D} \rightarrow \mathcal{A} b
$$

commutes with exact colimits, denoted here by $\stackrel{\text { lim, }}{\longrightarrow}$ if, for every directed system $\left(M_{\lambda}\right)_{\lambda \in \Lambda}$ of $\mathcal{O}_{X} G$-modules, the natural map

$$
\stackrel{\lim }{\longrightarrow} T\left(M_{\lambda}\right) \rightarrow T\left(\underline{\lim } M_{\lambda}\right)
$$

is an isomorphism. Analogously, we say a functor

$$
S: \mathfrak{M D D}_{\mathcal{X}} G \rightarrow \mathcal{A} b
$$


commutes with exact limits, denoted here by $\underset{\longleftarrow}{\longleftarrow}$, if, for every inverse system $\left(N_{\lambda}\right)_{\lambda \in \Lambda}$ of $\mathcal{O}_{x} G$-comodules, the natural map

$$
S\left(\underset{\lim }{\longleftarrow} N_{\lambda}\right) \rightarrow \underset{\lim }{\longleftarrow} S\left(N_{\lambda}\right)
$$

is an isomorphism.

We say an $\mathcal{O}_{X} G$-module $M(-)$ is finitely generated if there is a finitely generated free module mapping onto it. In particular, there is a $G$-finite $G$-set $\Delta$ such that $\mathbb{Z}[-, \Delta] \rightarrow M(-)$ (here we are extending the notation $\mathbb{Z}[-, \Delta]$ to non-transitive sets in the obvious way).

Lemma 5.1. Let $M$ be an $\mathcal{O}_{x} G$-module. Then $M$ is the direct colimit of its finitely generated submodules.

Proof. This follows from [15], §9.19.

The notions of type Bredon-FP, Bredon- $\mathrm{FP}_{n}$ and Bredon-FP $\infty$ are defined in terms of projective resolutions over $\mathcal{O}_{x} G$ analogously to the classical notions of type FP, $\mathrm{FP}_{n}$ and $\mathrm{FP}_{\infty}$.

Proposition 5.2. Let $A$ be an $\mathcal{O}_{x} G$-module of type Bredon- $\mathrm{FP}_{n}, 0 \leq n \leq \infty$. Then:

(i) For every exact limit, the natural homomorphism

$$
\operatorname{Tor}_{k}^{\mathfrak{x}}\left(\lim _{\longleftarrow} N_{*}, A\right) \rightarrow \underset{\lim }{\longleftarrow} \operatorname{Tor}_{k}^{\mathfrak{x}}\left(N_{*}, A\right)
$$

is an isomorphism for all $k \leq n-1$ and an epimorphism for $k=n$.

(ii) For every exact colimit, the natural homomorphism

$$
\stackrel{\lim }{\longrightarrow} \operatorname{Ext}_{\mathfrak{X}}^{k}\left(A, M_{*}\right) \rightarrow \operatorname{Ext}_{\mathfrak{X}}^{k}\left(A, \stackrel{\lim }{\longrightarrow} M_{*}\right)
$$

is an isomorphism for all $k \leq n-1$ and a monomorphism for $k=n$.

Proof. The proof goes completely analogously to that of Bieri [1], Proposition 1.2. It relies on the Yoneda isomorphisms, i.e., that $N \otimes_{\mathfrak{X}} \mathbb{Z}[-, G / K] \cong N(G / K)$ and $\operatorname{Hom}_{\mathfrak{X}}(\mathbb{Z}[-, G / K], M) \cong M(G / K)$, the fact that $\lim$ and $\operatorname{Hom}_{\mathfrak{X}}(-, M)$ commute with finite direct sums and that $\lim _{\longleftarrow}$ and $\lim _{\longrightarrow}$ are exact and hence commute with the homology functor.

Bieri's argument can be carried through completely for Bredon-Ext and BredonTor functors.

Theorem 5.3. Let $A$ be an $\mathcal{O}_{x} G$-module. Then the following are equivalent:

(i) A is of type Bredon- $\mathrm{FP}_{n}$. 
(ii) For every exact colimit, the natural homomorphism

$$
\stackrel{\lim }{\longrightarrow} \operatorname{Ext}_{\mathfrak{X}}^{k}\left(A, M_{*}\right) \rightarrow \operatorname{Ext}_{\mathfrak{X}}^{k}\left(A, \underset{\lim }{\longrightarrow} M_{*}\right)
$$

is an isomorphism for all $k \leq n-1$ and a monomorphism for $k=n$.

(iii) For the direct limit of any directed system of $\mathcal{O}_{x} G$-modules $M_{*}$ with $\underline{\lim } M_{*}=$ 0 , one has $\underset{\lim }{\longrightarrow} \operatorname{Ext}_{\mathfrak{X}}^{k}\left(A, M_{*}\right)=0$ for all $k \leq n$.

Proof. The implications (i) $\Longrightarrow$ (ii) $\Longrightarrow$ (iii) are either obvious or follow from Proposition 5.2. Every $\mathcal{O}_{x} G$-module is the directed colimit of finitely generated submodules, Lemma 5.1, and hence (iii) $\Longrightarrow$ (i) is proved completely analogously to [1], Theorem 1.3 (iiib) $\Longrightarrow$ (i).

Theorem 5.4. Let $A$ be an $\mathcal{O}_{x} G$-module. Then the following are equivalent:

(i) $A$ is of type Bredon- $\mathrm{FP}_{n}$.

(ii) For every exact limit, the natural homomorphism

$$
\operatorname{Tor}_{k}^{\mathfrak{x}}\left(\lim _{\longleftarrow} N_{*}, A\right) \rightarrow \underset{\longleftarrow}{\lim } \operatorname{Tor}_{k}^{\mathfrak{X}}\left(N_{*}, A\right)
$$

is an isomorphism for all $k \leq n-1$ and an epimorphism for $k=n$.

(iii) For any $K \in \mathfrak{X}$ consider any arbitrary direct product $\prod_{\Lambda_{K}} \mathbb{Z}[G / K,-]$. Then the natural map

$$
\operatorname{Tor}_{k}^{\mathfrak{X}}\left(\prod_{K \in \mathfrak{X}} \prod_{\Lambda_{K}} \mathbb{Z}[G / K,-], A\right) \rightarrow \prod_{K \in \mathfrak{X}} \prod_{\Lambda_{K}} \operatorname{Tor}_{k}^{\mathfrak{X}}(\mathbb{Z}[G / K,-], A)
$$

is an isomorphism for all $k \leq n-1$ and an epimorphism for $k=n$.

Proof. The implications (i) $\Longrightarrow$ (ii) $\Longrightarrow$ (iii) are again either obvious or consequence of Proposition 5.2.

(iii) $\Longrightarrow$ (i): The proof is in the same spirit as Bieri's proof. We begin by letting $n=0$ and claim that $A$ is finitely generated as an $\mathcal{O}_{x} G$-module. As an index set we take $\prod_{K \in \mathfrak{X}} A(G / K)$ and consider $\prod_{K \in \mathfrak{X}} \prod_{a \in A(G / K)} \mathbb{Z}[G / K,-]$. By (iii), the natural map

$$
\mu:\left(\prod_{K \in \mathfrak{X}} \prod_{A(G / K)} \mathbb{Z}[G / K,-]\right) \otimes \mathfrak{X} A(-) \rightarrow \prod_{A(G / K)} A(G / K)
$$

is an epimorphism. Let $c$ be the element with $\mu(c)=\prod_{K \in \mathfrak{X}} \prod_{a \in A(G / K)} a$. Then $c$ is of the form

$$
c=\sum_{i=1}^{l}\left(\prod_{K \in \mathfrak{X}} \prod_{A(G / K)} f_{i}^{a, K}\right) \otimes b_{i}
$$

for certain subgroups $H_{1}, \ldots, H_{l} \in \mathfrak{X}$ and elements $b_{i} \in A\left(G / H_{i}\right)$. Here, $f_{i}^{a, K} \in$ $\mathbb{Z}\left[G / K, G / H_{i}\right]$. Now we claim that there is an epimorphism

$$
\tau: \bigoplus_{i=1}^{l} \mathbb{Z}\left[-, G / H_{i}\right] \rightarrow A
$$


given by $\tau(f):=f^{*}\left(b_{i}\right) \in A(G / K)$ whenever $f \in \mathbb{Z}\left[G / K, G / H_{i}\right]$. Observe that this is well defined. In particular it is functorial. To prove the claim, take any $K \in \mathfrak{X}$ and any $a \in A(G / K)$. Note that

$$
\mu(c)=\sum_{i=1}^{l} \prod_{K \in \mathfrak{X}} \prod_{a \in A(G / K)}\left(f_{i}^{a, K}\right)^{*}\left(b_{i}\right)=\prod_{K \in \mathfrak{X}} \prod_{a \in A(G / K)} \sum_{i=1}^{l}\left(f_{i}^{a, K}\right)^{*}\left(b_{i}\right)
$$

so the fact that $c$ maps onto the diagonal means that

$$
a=\sum_{i=1}^{l}\left(f_{i}^{a, K}\right)^{*}\left(b_{i}\right)=\tau\left(\sum_{i=1}^{l} f_{i}^{a, K}\right) .
$$

The case $n \geq 1$ is now done analogously to [1], Theorem 1.3, using a diagram chase.

Remark 5.5. For $n \geq 1$, condition (iii) is equivalent to the following, which in ordinary homology is often referred to as the Bieri-Eckmann criterion for $\mathrm{FP}_{n}$ : For every subgroup $K \in \mathfrak{X}$ consider an arbitrary direct product $\prod_{\Lambda_{K}} \mathbb{Z}[G / K,-]$. Then the natural map

$$
\left(\prod_{K \in \mathfrak{X}} \prod_{\Lambda_{K}} \mathbb{Z}[G / K,-]\right) \otimes \mathfrak{X} A(-) \rightarrow \prod_{K \in \mathfrak{X}} \prod_{\Lambda_{K}} A(G / K)
$$

is an isomorphism and $\operatorname{Tor}_{k}^{\mathfrak{X}}\left(\prod_{K \in \mathfrak{X}} \prod_{\Lambda_{K}} \mathbb{Z}[G / K,-], A\right)=0$, for all $1 \leq k \leq n-1$. We call this condition the global Bieri-Eckmann criterion for Bredon homology.

We say a group satisfies the local Bieri-Eckmann criterion for Bredon cohomology if, for any $K$ and direct product as before, the natural map

$$
\left(\prod_{\Lambda_{K}} \mathbb{Z}[G / K,-]\right) \otimes \mathfrak{X} A(-) \rightarrow \prod_{\Lambda_{K}} A(G / K)
$$

is an isomorphism and $\operatorname{Tor}_{k}^{\mathfrak{X}}\left(\prod_{\Lambda_{K}} \mathbb{Z}[G / K,-], A\right)=0$ for all $1 \leq k \leq n-1$.

\section{Classifying spaces with finite isotropy}

In this section we shall restrict ourselves to the family $\mathcal{F}$ of all the finite subgroups of $G$.

To stay in line with notation previously used, we say a module is of type $\underline{\mathrm{FP}}_{\infty}$ if it is of type Bredon-FP ${ }_{\infty}$ with respect to $\mathscr{F}$. The notions of $\underline{\mathrm{FP}}_{n}$ and $\underline{\mathrm{FP}}$ are defined analogously. For Bredon cohomology with respect to $\mathcal{F}$ there is a good algebraic description for modules of type $\underline{\mathrm{FP}}_{n}$. For the original approach via classifying spaces, see [16].

Theorem 6.1 ([13]). Let $G$ be a group having finitely many conjugacy classes of finite subgroups. Then an $\mathcal{O}_{\mathcal{F}} G$-module $M(-)$ is of type $\underline{\mathrm{FP}}_{n}$ if and only if $M(G / K)$ is of type $\mathrm{FP}_{n}$ as a $\mathbb{Z}(W K)$-module for each finite subgroup $K$ of $G$. 
It was also shown ([13]) that a group $G$ is of type $\underline{\mathrm{FP}}_{0}$ if and only if $G$ has finitely many conjugacy classes of finite subgroups. Hence we have the following corollary:

Corollary 6.2 ([13]). A group $G$ is of type ${\underline{\mathrm{FP}_{n}}}_{n}$ if and only if $G$ has finitely many conjugacy classes of finite subgroups and $C_{G}(K)$ is of type $\mathrm{FP}_{n}$ for every finite subgroup $K$ of $G$.

Recall that we say a group $G$ is of Bredon type $\mathrm{FP}_{n}$ if the trivial module $\mathbb{Z}(-)$ is of type $\mathrm{FP}_{n}$ as an $\mathcal{O}_{x} G$-module. We can, of course rephrase Theorems 5.3 and 5.4 in terms of Bredon cohomology and Bredon homology replacing the module $A(-)$ with $\mathbb{Z}(-), \operatorname{Ext}_{\mathfrak{X}}^{*}(A,-)$ with $\mathrm{H}_{\mathfrak{X}}^{*}(G,-)$ and $\operatorname{Tor}_{*}^{\mathfrak{X}}(-, A)$ with $\mathrm{H}_{*}^{\mathfrak{X}}(G,-)$.

We shall now weaken the hypothesis on the conjugacy classes of finite subgroups.

Definition 6.3. We say a group is of type quasi-FP $\underline{\mathrm{FP}}_{n}$ if, for each finite subgroup $K$ of $G$ there are finitely many conjugacy classes of subgroups isomorphic to $K$ and the Weyl groups $W K:=N_{G}(K) / K$ are of type $\mathrm{FP}_{n}$.

Note that a group of type quasi-FP $\underline{\mathrm{F}}_{n}$ with a bound on the orders of the finite subgroups is of type $\underline{\mathrm{FP}}_{n}$.

Let $k$ be a positive integer. We denote by $\mathbb{Z}_{k}(-)$ the $\mathcal{O}_{\mathscr{F}} G$-module defined by

$$
\mathbb{Z}_{k}(G / H)= \begin{cases}\mathbb{Z} & \text { if }|H| \leq k \\ 0 & \text { otherwise }\end{cases}
$$

together with the obvious morphisms.

Lemma 6.4. A group $G$ is of type quasi- $\underline{\mathrm{FP}}_{0}$ if and only if, for each $k \geq 1$, the module $\mathbb{Z}_{k}(-)$ is finitely generated. Moreover, in that case, the finite $G$-set $\Delta_{k}$ with $\mathbb{Z}\left[-, \Delta_{k}\right] \rightarrow \mathbb{Z}_{k}(-)$ can be chosen to have stabilisers of order bounded by $k$.

Proof. Suppose that $G$ is of type quasi-FP $\underline{-F}_{0}$. Take

$$
\Delta_{k}=\bigsqcup_{|H| \leq k, \text { up to } G \text {-conj. }} G / H .
$$

This is a $G$-finite $G$-set with stabilisers of order bounded by $k$ and $\mathbb{Z}\left[-, \Delta_{k}\right] \rightarrow$ $\mathbb{Z}_{k}(-)$.

For the converse, we need to show that, for each finite subgroup $K$, there are only finitely many conjugacy classes of subgroups of order bounded by $k=|K|$. Let $\Delta_{k}$ be the finite $G$-set with $\mathbb{Z}\left[-, \Delta_{k}\right] \rightarrow \mathbb{Z}_{k}(-)$ and take any finite subgroup $H$ of $G$ with $|H| \leq k$. Hence $\mathbb{Z}_{k}(G / H) \cong \mathbb{Z} \neq 0$. Since the map $\mathbb{Z}[G / H, \Delta] \rightarrow \mathbb{Z}_{k}(G / H)$ is onto, it follows that $\mathbb{Z}[G / H, \Delta] \neq 0$ and hence $H$ has to be subconjugated to one of the finitely many stabilisers of $\Delta$. 
Note that finitely generated $\mathcal{O}_{\mathscr{F}} G$-modules are precisely those of type $\underline{\mathrm{FP}}_{0}$. Fix an integer $k \geq 1$ and let $M_{k}(-)$ be an $\mathcal{O}_{\mathscr{F}} G$-module such that $M_{k}(G / L)=0$ whenever $|L|>k$. Suppose that $M_{k}(-)$ is finitely generated. Then there exists a $G$-finite $G$-set $\Delta$ with stabilisers of order $\leq k$ and a short exact sequence of $\mathcal{O}_{\mathscr{F}} G$-modules

$$
N_{k}(-) \succ \mathbb{Z}[-, \Delta] \rightarrow M_{k}(-)
$$

with the property that $N_{k}(G / L)=0$ for all finite subgroups $L$ with $|L|>k$.

Proposition 6.5. A group $G$ is of type quasi- $\underline{\mathrm{FP}}_{n}$ if and only if the $\mathcal{O}_{\mathcal{F}} G$-module $\mathbb{Z}_{k}(-)$ is of type $\underline{\mathrm{FP}}_{n}$ for each integer $k \geq 1$.

Proof. The "if"-direction follows from Lemma 6.4, Theorem 6.1, and the definition as $\mathbb{Z}_{|K|}(G / H)$ is of type $\mathrm{FP}_{n}$ as a $W H$-module for each $|H| \leq|K|$.

Now suppose that $G$ is of type quasi- $\underline{\mathrm{FP}}_{n}$. For each $k \geq 1$ we construct a projective resolution of $\mathbb{Z}_{k}(-)$ which is finitely generated in dimensions up to $n$; note that we may assume $n>0$. By Lemma 6.4 and the above remark we have a short exact sequence

$$
C_{0}(-) \succ \mathbb{Z}\left[-, \Delta_{0}\right] \rightarrow \mathbb{Z}_{k}(-)
$$

with $\Delta_{0}$ a $G$-finite $G$-set and $C_{0}(G / L)=0$ for all $|L|>k$. We claim that $C_{0}(-)$ is a finitely generated $\mathcal{O}_{\mathcal{F}} G$-module.

We know that there are finitely many conjugacy classes of subgroups of order bounded by $k$. Let $H$ be one of those. As $\Delta_{0}$ is $G$-finite, the $W H$-module $\mathbb{Z}\left[G / H, \Delta_{0}\right]$ is of type $\mathrm{FP}_{\infty}$. This is a consequence of the fact that for any $K$, $\mathbb{Z}[G / H, G / K]$ is a sum of exactly $\left|\left\{x \in N_{G}(H) \backslash G / K \mid H^{x^{-1}} \leq K\right\}\right| W H$ modules, which are of type $\mathrm{FP}_{\infty}$. As $K$ is finite, this sum must also be finite. So evaluating the previous short exact sequence at $G / H$, we see that the $W H$-module $C_{0}(G / H)$ is of type $\mathrm{FP}_{n-1}$ and in particular, finitely generated. Fix a finite $W H$ generating set $X_{H}$ for $C_{0}(G / H)$. Then the $\mathcal{O}_{\mathcal{F}} G$-set formed by the union of all those $X_{H}$ where $H \in \operatorname{Stab} \Delta_{0}$, generates $C_{0}$.

We can now proceed to construct the desired resolution by using the remark before Proposition 6.5.

Theorem 6.6. Let $G$ be of type quasi- $\underline{\mathrm{FP}}_{n}$, where $n \geq 1$. Then $G$ satisfies the local Bieri-Eckmann criterion for Bredon homology.

Proof. It follows from the definition of the modules $\mathbb{Z}_{k}(-)$ that

$$
\mathbb{Z}(-)=\underset{k \in \mathbb{N}}{\underset{\lim }{\longrightarrow}} \mathbb{Z}_{k}(-)
$$

In the category of $\mathcal{O}_{\mathscr{F}} G$-modules the construction of a free module mapping onto a given one is functorial. Hence, we can get a direct colimit of free resolutions 
Bredon cohomological finiteness conditions for generalisations of Thompson groups 955

$\lim _{k \in \mathbb{N}}\left(F_{*, k}(-) \rightarrow \mathbb{Z}_{k}(-)\right)=F_{*}(-) \rightarrow \mathbb{Z}(-)$, which gives us a flat resolution of $\overrightarrow{\mathbb{Z}(-)}$. For details the reader is referred to [22], Lemma 3.4. Hence

$$
\begin{aligned}
\mathrm{H}_{k}^{\mathcal{F}}\left(G, \prod_{\Lambda} \mathbb{Z}[G / K,-]\right) & =\mathrm{H}_{k}\left(\prod_{\Lambda} \mathbb{Z}[G / K,-] \otimes_{\mathcal{F}} F_{*}(-)\right) \\
& =\mathrm{H}_{k}\left(\prod_{\Lambda} \mathbb{Z}[G / K,-] \otimes_{\mathcal{F}} \lim _{k \in \mathbb{N}} F_{*, k}(-)\right) \\
& =\underset{k \in \mathbb{N}}{\lim _{k}} \mathrm{H}_{k}\left(\prod_{\Lambda} \mathbb{Z}[G / K,-] \otimes_{\mathcal{F}} F_{*, k}(-)\right) \\
& =\underset{k \in \mathbb{N}}{\lim _{k}} \operatorname{Tor}_{k}\left(\prod_{\Lambda} \mathbb{Z}[G / K,-], \mathbb{Z}_{k}(-)\right)=0,
\end{aligned}
$$

where the last line follows from Proposition 6.5 and Theorem 5.4. The first assertion follows by a similar argument.

For each $k \geq 1$ we consider the family $\mathscr{F}_{k}$ and the orbit category $\mathcal{O}_{\mathcal{F}_{k}} G$. For a given positive integer $k$ the family $\widetilde{F}_{k}$ consists of all subgroups $H$ of $G$ with $|H| \leq k$. By using the arguments of the proofs of Lemma 6.4 and Proposition 6.5 we can show:

Proposition 6.7. A group is of type quasi- $\underline{\mathrm{FP}}_{n}$ if and only if it is of type Bredon- $\mathrm{FP}_{n}$ over $\mathcal{O}_{\mathcal{F}_{k}} G$ for each $k$.

We can also rephrase Theorems and 5.3 and 5.4:

Corollary 6.8. Let $G$ be a group. Then the following are equivalent:

(i) $G$ is of type quasi-FP $\underline{\mathrm{F}}_{n}$.

(ii) For every exact colimit and any $k$, the natural homomorphism

$$
\stackrel{\lim }{\longrightarrow} \mathrm{H}_{\mathfrak{F}_{k}}^{l}\left(G, M_{*}\right) \rightarrow \mathrm{H}_{\mathfrak{F}_{k}}^{l}\left(G, \lim _{\longrightarrow} M_{*}\right)
$$

is an isomorphism for all $l \leq n-1$, and a monomorphism for $l=n$.

(iii) For any $k$ and any $K \in \mathfrak{F}_{k}$ consider an arbitrary direct product $\prod_{\Lambda_{K}} \mathbb{Z}[G / K,-]$. Then the natural map

$$
\mathrm{H}_{l}^{\mathfrak{F} k}\left(\prod_{K \in \mathfrak{F}_{k}} \prod_{\Lambda_{K}} \mathbb{Z}[G / K,-], A\right) \rightarrow \prod_{K \in \mathfrak{F}_{k}} \prod_{\Lambda_{K}} \mathrm{H}_{l}^{\mathfrak{F} k}(\mathbb{Z}[G / K,-], A)
$$

is an isomorphism for all $l \leq n-1$ and an epimorphism for $l=n$.

One may also add the statements analogous to Theorems 5.3 (ii) and 5.4 (ii). Note also that for $n \geq 1$ the above is equivalent to:

(iv) For any $k$, any $K \in \mathfrak{F}_{k}$ and any arbitrary direct product $\prod_{\Lambda_{K}} \mathbb{Z}[G / K,-]$, the natural map

$$
\mathbb{Z}_{k}(-) \otimes_{\mathfrak{F}_{k}} \prod_{K \in \mathfrak{F}_{k}} \prod_{\Lambda_{K}} \mathbb{Z}[G / H,-] \rightarrow \prod_{K \in \mathfrak{F}_{k}} \prod_{\Lambda_{K}} \mathbb{Z}_{k}
$$

is an isomorphism and $\mathrm{H}_{l}^{\mathfrak{F}_{k}}\left(G, \prod_{K \in \mathfrak{F}_{k}} \prod_{\Lambda_{K}} \mathbb{Z}[G / H,-]\right)=0$ for all $1 \leq l \leq$ $n-1$. 
Definition 6.9. We say a group $G$ is of type quasi- $\underline{F}_{\infty}$ if for all positive integers $k$, $G$ admits a finite type model for $E_{\mathfrak{F}_{k}} G$.

Analogously to the algebraic case, any group of type quasi- $\underline{F}_{\infty}$, which has a bound on the orders of the finite subgroups, is of type $\underline{F}_{\infty}$.

Lück's Theorem [16], Theorem 4.2 (Theorem 1.2) goes through for arbitrary families of finite subgroups. Hence combining Theorems 1.1 and 1.2 yields:

Proposition 6.10. A group $G$ is of type quasi- $\underline{\mathrm{F}}_{\infty}$ if and only if $G$ is of type quasi-跙 and $G$ and all centralisers $C_{G}(K)$ of finite subgroups are finitely presented.

We can now prove what is largely equivalent to Proposition 6.7:

Theorem 6.11. A group $G$ is of type quasi- $\mathrm{F}_{\infty}$ if and only if it admits a model for $\underline{E} G$, which is the mapping telescope of finite type models for $E_{\mathfrak{F}_{k}} G$ for each $K \in \mathcal{F}$.

Proof. The "if"-direction follows directly from the definition. Now suppose we have finite type models $X_{K}$ for $E_{\mathfrak{F}_{k}} G$ for all $K \in \mathscr{F}$. For each $H \leq K$ the universal property for classifying spaces for a family yields $G$-maps $v_{H}^{K}: X_{H} \rightarrow X_{K}$. Now the mapping telescope yields a $G$-CW-complex X, for which $X^{K}$ is contractible for all $K \in \mathscr{F}$ and empty otherwise.

\section{Bredon cohomological finiteness properties for generalised Higman-Thompson groups}

We can now prove

Theorem 7.1. Let $U_{r}(\Sigma)$ be a Cantor algebra with order-preserving $\Sigma$. Then the following conditions are equivalent for $1 \leq r \leq d$ :

(i) $T_{r}(\Sigma)$ is quasi- $\underline{\mathrm{FP}}_{\infty}$.

(ii) $T_{l}(\Sigma)$ is of (ordinary) type $\mathrm{FP}_{\infty}$ for any $1 \leq l \leq d$ such that $\operatorname{gcd}(l, d) \mid r$.

Proof. Assume that (i) holds and take $1 \leq l \leq d$ with $\operatorname{gcd}(l, d) \mid r$. This condition implies that there is some positive integer $w$ with $l w \equiv r \bmod d$. Then we may choose an admissible subset $A \subseteq U_{r}(\Sigma)$ of cardinality $l w$ and consider the subgroup $Q$ of $T_{r}(\Sigma)$ defined by cyclic permutations of $A$ on $l$ orbits all of length $w$. By Theorem 4.8, $C_{T_{r}(\Sigma)}(Q)$ is an extension of a finite group by $T_{l}(\Sigma)$ so this last group must be of type $\mathrm{FP}_{\infty}$.

Now assume (ii). Observe that for any finite subgroup $Q$ of cardinality $w$ and any admissible subset $Y_{1}$ fixed by $Q$, the observation before Theorem 4.8 implies that $\left|Y_{1}\right|=l w \equiv r \bmod d$ for certain $l$, thus $\operatorname{gcd}(l, d) \mid d r$. This together with Theorem 4.8 implies that $T_{r}(\Sigma)$ is quasi- $\underline{\mathrm{FP}}_{\infty}$. 
We also have the same result for finiteness conditions on classifying spaces.

Theorem 7.2. Let $U_{r}(\Sigma)$ be a Cantor algebra with order-preserving $\Sigma$. Then the following conditions are equivalent:

(i) $T_{r}(\Sigma)$ is quasi- $\underline{\mathrm{F}}_{\infty}$.

(ii) $T_{l}(\Sigma)$ is of (ordinary) type $\mathrm{F}_{\infty}$ for any $1 \leq l \leq d$ such that $\operatorname{gcd}(l, d) \mid r$.

Proof. This follows from Theorem 4.8 exactly as Theorem 7.1.

Corollary 7.3. Let $U_{r}(\Sigma)$ be a Higman algebra. Then $T_{n, r}=T_{r}(\Sigma)$ is quasi- $\underline{\mathrm{F}}_{\infty}$.

Proof. This follows directly from [5] and Theorem 7.2.

Corollary 7.4. Let $U_{r}(\Sigma)$ be a Brown-Stein algebra. Then $T=T_{r}(\Sigma)$ is quasi-歼 ${ }_{\infty}$.

Proof. This is a consequence of Theorem 7.2 and [24], Theorem 2.5, where it is proven that $F_{r}(\Sigma)$ is finitely presented and of type $\mathrm{FP}_{\infty}$ for any $r$. Stein's argument carries over to $G$ and $T$, see [24].

Conjecture 7.5. Let $U_{r}(\Sigma)$ be a Cantor-algebra. Then:

(i) $G_{r}(\Sigma)$ is quasi- $\underline{\mathrm{FP}}_{\infty}$ if and only if $G_{l}(\Sigma)$ is of (ordinary) type $\mathrm{FP}_{\infty}$ for any $1 \leq l \leq d$.

(ii) $G_{r}(\Sigma)$ is quasi $-\underline{\mathrm{F}}_{\infty}$ if and only if $G_{l}(\Sigma)$ is of (ordinary) type $\mathrm{F}_{\infty}$ for any $1 \leq$ $l \leq d$.

Remark 7.6. Our description of centralisers of finite subgroups implies the "only if" part of this conjecture. To see this, assume that $G_{r}(\Sigma)$ is of type quasi-FP $\infty$ and choose for any $1 \leq l \leq d$ positive integers $n, s$ with $n \geq 3$ such that $l n+s \equiv r$ mod $d$. Then there is some admissible subset $Y \in \mathfrak{A}_{r}(\Sigma)$ of cardinality precisely $l n+s$ and we may consider the finite group $Q \cong S_{n} \leq G_{r}(\Sigma)$ defined by the action on $l$ orbits of $n$ elements as the natural representation of $S_{n}$ and acting trivially on the remaining $s$ elements of $Y$. Then Theorem 4.4 implies, using the same notation here, that

$$
C_{G_{r}(\Sigma)}(Q) \cong H_{l} \times H_{s} .
$$

Hence both $H_{l}$ and $H_{s}$ are of type $\mathrm{FP}_{\infty}$. Moreover, $H_{l}$ is an extension

$$
K_{1} \succ H_{l} \rightarrow G_{l}(\Sigma),
$$

where by Remark $4.7 K_{1}$ is a direct limit of products of $L=C_{S_{n}}\left(S_{n}\right)=1$. Thus, in this case, $L=K_{1}=1$ implying that $G_{l}(\Sigma)$ is of type $\mathrm{FP}_{\infty}$.

Remark 7.7. By [5], Proposition 4.1, $F_{n, r} \cong F_{n, s}$, for any $r, s$. However, this is false for the groups $G$, in fact $G_{n, r} \cong G_{n, s}$ implies that $\operatorname{gcd}(n-1, r)=\operatorname{gcd}(n-1, s)$ ([10], Theorem 6.4). Recently, Pardo [23] observed that the converse also holds true (see also [8]). 


\section{References}

[1] R. Bieri, Homological dimension of discrete groups. 2nd ed., Queen Mary College Mathematical Notes, Queen Mary College Department of Pure Mathematics, London 1981. Zbl 0357.20027 MR 715779

[2] R. Bieri and B. Renz, Valuations on free resolutions and higher geometric invariants of groups. Comment. Math. Helv. 63 (1988), 464-497. Zbl 0654.20029 MR 960770

[3] C. Bleak, H. Bowman, A. Gordon, G. Graham, J. Hughes, F. Matucci, and E. Sapir, Centralizers in the R. Thompson group $V_{n}$. Groups Geom. Dyn. 7 (2013), 821-865.

[4] M. G. Brin, Higher dimensional Thompson groups. Geom. Dedicata 108 (2004), 163-192. Zbl 1136.20025 MR 2112673

[5] K. S. Brown, K. S. Brown, Finiteness properties of groups. J. Pure Appl. Algebra 44 (1987), 45-75. Zbl 0613.20033 MR 885095

[6] J. Burillo and S. Cleary, Metric properties of higher-dimensional Thompson's groups. Pacific J. Math. 248 (2010), 49-62. Zbl 1236.20045 MR 2734164

[7] P. M. Cohn, Universal algebra. Math. Appl. 6, 2nd ed., D. Reidel Publishing Co., Dordrecht 1981. Zbl 0461.08001 MR 620952

[8] W. Dicks and C. Martínez-Pérez, Isomorphisms of Brin-Higman-Thompson groups. Israel J. Math., to appear. Doi 10.1007/s11856-013-0042-7

[9] J. Hennig and F. Matucci, Presentations for the higher-dimensional Thompson groups. Pacific J. Math. 257 (2012), 53-74. Zbl 1248.20035 MR 2948458

[10] G. Higman, Finitely presented infinite simple groups. Notes on Pure Mathematics 8, Australian National University, Canberra 1974. MR 0376874

[11] D. H. Kochloukova, C. Martínez-Pérez, and B. E. A. Nucinkis, Fixed points of finite groups acting on generalised Thompson groups. Israel J. Math. 187 (2012), 167-192. Zbl 06108418 MR 2891703

[12] D. H. Kochloukova, C. Martínez-Pérez, and B. E. A. Nucinkis, Cohomological finiteness properties of the Brin-Thompson-Higman groups $2 \mathrm{~V}$ and $3 \mathrm{~V}$. Proc. Edinburgh Math. Soc. (2) 56 (2013), 777-804. MR 3109758

[13] P. H. Kropholler, C. Martinez-Pérez, and B. E. A. Nucinkis, Cohomological finiteness conditions for elementary amenable groups. J. Reine Angew. Math. 637 (2009), 49-62. Zbl 1202.20055 MR 25981

[14] I. J. Leary and B. E. A. Nucinkis, Some groups of type VF. Invent. Math. 151 (2003), 135-165. Zbl 1032.20035 MR 1943744

[15] W. Lück, Transformation groups and algebraic K-theory. Lecture Notes in Math. 1408, Springer-Verlag, Berlin 1989. Zbl 0679.57022 MR 1027600

[16] W. Lück, The type of the classifying space for a family of subgroups. J. Pure Appl. Algebra 149 (2000), 177-203. Zbl 0955.55009 MR 1757730

[17] W. Lück and D. Meintrup, On the universal space for group actions with compact isotropy. In Geometry and topology: Aarhus (1998), Contemp. Math. 258, Amer. Math. Soc., Providence, RI 2000, 293-305. Zbl 0979.55010 MR 1778113 
[18] C. Martínez-Pérez, F. Matucci and B. E. A. Nucinkis, Cohomological finiteness conditions and centralisers in generalisations of Thompson's group V. Preprint, arXiv:1309.7858 [math.GR].

[19] F. Matucci, Algorithms and classification in groups of piecewise-linear homeomorphisms. Ph.D. thesis, Cornell University, Ithaca 2008. Preprint, arXiv:0807.2871 [math.GR].

[20] D. Meintrup and T. Schick, A model for the universal space for proper actions of a hyperbolic group. New York J. Math. 8 (2002), 1-7. Zbl 0990.20027 MR 1887695

[21] G. Mislin, Equivariant K-homology of the classifying space for proper actions. In Proper group actions and the Baum-Connes conjecture, Notes on an Advanced Course on Proper Group Actions, Adv. Courses Math. CRM Barcelona, Birkhäuser, Basel 2003, 1-78. Zbl 01961971 MR 2027169

[22] B. E. A. Nucinkis, On dimensions in Bredon homology. Homology Homotopy Appl. 6 (2004), 33-47. Zbl 1068.20054 MR 2061566

[23] E. Pardo, The isomorphism problem for Higman-Thompson groups. J. Algebra 344 (2011), 172-183. Zbl 1247.20034 MR 2831934

[24] M. Stein, Groups of piecewise linear homeomorphisms. Trans. Amer. Math. Soc. 332 (1992), 477-514. Zbl 0798.20025 MR 1094555

[25] K. Vogtmann, Automorphisms of free groups and outer space. Geom. Dedicata 94 (2002), 1-31. Zbl 1017.20035 MR 1950871

Received May 3, 2011; revised March 8, 2012

C. Martínez-Pérez, Departamento de Matemáticas, Universidad de Zaragoza, 50009 Zaragoza, Spain

E-mail: conmar@unizar.es

B. E. A. Nucinkis, Department of Mathematics, Royal Holloway, University of London, Egham, TW20 0EX, UK

E-mail: brita.nucinkis@rhul.ac.uk 